\title{
The more we know, the less we agree: public announcements and higher-order expectations*
}

\author{
Péter Kondor \\ FMG - London School of Economics and Political Science
}

December 2004

\begin{abstract}
The stylized fact that public announcements in financial markets are followed by intense trading, high trading volume and volatile prices, is widely perceived as the sign of increasing disagreement due to the announcement. However, it is common to argue that this would be inconsistent with Bayesian-learning and common priors. In this paper, we not only show that - with certain information structures - increasing disagreement is possible in a Bayesian model, but we also argue that with the assumption that traders trade for resale - so they try to second guess future traders' guesses - there are information structures which are simple, intuitive and plausible and result in increasing disagreement even in a standard, multi-period Grossman-Stiglitz model.

Keywords: confirmatory bias, public announcements, trading volume, higher-order expectations, short-term traders.
\end{abstract}

JEL classification: D82, D84, G11, G12.

\section{Introduction}

It is a well established stylized fact in financial markets that public announcements are followed by intense trading, high trading volume and volatile prices. This is widely perceived as the sign that the public announcement increases disagreement, and the polarized asset valuations are channeled into prices by the hectic trading activity. (Evans and Lyons, 2003 Love and Payne,2003, Fleming and Remolona,1999, Bamber et al, 1997, Kim and Verrecchia 1997, 1994, 1991, Kandel and Pearson, 1995, Harris and Raviv, 1993, Varian, 1989). It is also common to argue that agents' different reaction to the same public information cannot be explained with a standard Bayesian-learning model with common priors. Varian (1989), Harris and Raviv (1993) and Kandel and Pearson (1995) assume that agents interpret the same information differently, because they have different priors, while Evans and Lyons (2003) and Kim and Verrecchia $(1997,1993)$ suggest a model where the announcement incorporates a private information element i.e. agents look at the same piece of information but they see something

${ }^{*}$ E-mail address: p.kondor@lse.ac.uk. We are grateful for the help received from Gabrielle Demange, Bernardo Guimaraes, Hyun Song Shin, Gergely Ujhelyi, Dimitri Vayanos and seminar participants at the LSE and at IE-HAS. All remaining errors are our own. We gratefully acknowledge the EU grant "Archimedes Prize" ( HPAW-CT-2002-80054). 
different. In behavioral economics similar phenomena ${ }^{1}$ are explained by the so-called confirmatory bias: each agent tends to interpret the same information in a way that supports his or her own view. Rabin and Schrag (1999) put it as follows:

"... a large and growing body of psychological research suggests that the way people process information often departs systematically from Bayesian updating. In this paper we formally model and explore the consequences of one particular departure from Bayesian rationality: confirmatory bias. [...] The most striking evidence for the confirmatory bias is a series of experiments demonstrating how providing the same ambiguous information to people who differ in their initial beliefs on some topic can move their beliefs farther apart." Rabin and Schrag(1999, pp 38,43.)

In this paper, we show that with certain information structures a public announcement can increase disagreement even with standard Bayesian decision makers and common priors. More importantly, we argue that with early traders who buy for resale - i.e. who trade on their expectation of the expectation of future traders - there are such information structures for financial markets which are simple, intuitive and plausible. Hence, we present a Grossman-Stiglitz type standard rational expectation model with higher-order expectations, where public announcements increase disagreement and hence generate large trading volume. We also show that our model is consistent with recent evidence - observed in high-frequency data sets from foreign exchange and fixed income markets - that although at the time of the announcement there is an initial price adjustment, it is followed by a prolonged period with increased, more volatile and more informative trading (Evans and Lyons, 2003 Love and Payne,2003, Fleming and Remolona, 1999, Love 2004). There is also some direct evidence from equity markets (Bamber et al, 1997), which uses analysts forecast as a proxy for traders opinion, that in line with our model shows that the increased trading after announcements is indeed associated with increasing disagreement.

The intuition behind why expectations on expectations of others i.e. higher-order expectations can be useful to explain increasing disagreement is simple. In the interesting cases of increasing disagreement, agents adjust their beliefs differently after the public announcement: the same information will be good news for one agent and bad news for an other agent. However, it is quite a natural intuitive requirement to assume that good public signals should be associated with strong fundamentals and vice versa, regardless of the private information. The twist in our model is that our early traders will not be interested in the true value, they will be interested in the opinion of future traders about the true value. Hence, high public signals can continue to be associated with strong fundamentals; it is enough if the same public signal with a particular private piece of information is associated with a low average private signal of future traders while together with other private signals it is associated with a high average private signal of future traders. We show that precisely this will be the case, if the connection between private information sets of agents in different periods is not too strong, but

\footnotetext{
${ }^{1}$ In a typical experiment (conducted by Lord, Ross, and Lepper in 1979) two groups of people were given a sequence of studies on the merits of capital punishment as a deterrent to crime. Individuals in different groups had opposite initial opinion about the issue. After seeing exactly the same information, both groups got even more convinced of their initial opinion.
} 
the public signal is related to all agents' private information. In this case the public information will connect early traders' private information to future traders private information. Therefore, before the public announcement, there will not be too much dispersion among differently informed early traders' guesses on late comers average guess, because early traders' private information will not be very informative on late comers' information. However, at the moment of the public announcement, their private information gets connected, so informational differences among early traders spread out their asset valuation as well. Hence, it is pre-existing private information which becomes relevant in guessing future traders expectation due to the announcement. As trades are driven by differences in information, this newly relevant information differential is channeled into prices by increased trading activity. Different traders see the same public signal: it just gives significance to their pre-existing private information, and makes them trade on it.

Our model fits well with the recent flow of applied theoretical papers analyzing the effect of higherorder expectations on financial markets ${ }^{2}$. The leading metaphor in this literature is the famous beauty contest example of Keynes which compares speculative trading to those beauty contests where gifts are distributed among those who voted for the winner. Similar to the metaphor with the contestants, the problem of speculative traders is to choose those assets which future traders will consider valuable - so the resale price will be high -, which does not necessarily coincide with those that they consider undervalued. The main observation of these papers is that higher-order expectations in asymmetric information environments may behave very differently to first-order expectations: the law of iterated expectations is violated in a systematic way. This fact in turn, can explain stylized facts of financial markets in a novel way. The first paper in this literature - and the most closely related to our work - is Allen, Morris and Shin (2003), which shows that assuming short-horizon traders in the standard dynamic asymmetric information model of Brown and Jennings (1989) implies that prices will be oversensitive to public information in the early periods, because higher-order expectations overreacts the public signal. In the companion paper, Kondor (2004) - similar to this model - allows for an information structure, where private information sets of early traders are less connected. As a consequence, expectations on resale price (higher-order expectations) can move against expectations on fundamental value (first-order expectations) as traders' private information changes. Hence, early traders - expecting high resale price of low-value assets - will buy overvalued assets and sell undervalued assets even if future traders are rational. In the current paper, we concentrate on second moments. The driving force is the fact that dispersion of higher-order expectations can increase after an announcement even when dispersion of first-order expectations decreases.

Our work is naturally related to the theoretical work on trading volume and public information releases. The literature can be divided into two groups. Bayesian models with common priors (Brown and Jennings, 1989, Kim and Verrecchia, 1991, He and Wang, 1995) do not deliver disagreement increasing announcements, consequently they have problems explaining the empirical regularities. The basic structure of these models are nested in our set up, so we will be able to point out the

\footnotetext{
${ }^{2}$ This literature originates from the application of results from the global games literature on currency crisis by Morris and Shin (1998), but recently it has been extended to non-global games environments. In particular, monetary economics seems to be a fruitful area in the higher-order expectations literature (see Woodford, 2001, Hellwig 2002, Adams, 2003, Amato and Shin 2003).
} 
difference which makes our model capable of explaining high trading volume around announcement. In contrast, models with heterogenous priors and/or non-Bayesian agents in contrast (Varian, 1986, Harris and Raviv, 1993, Kandel and Pearson, 1995, Evans and Lyons, 2001) can produce disagreement and volume, but at the expense of assuming non-common priors or different perception of the same public information. They argue that as standard models are inherently incapable of explaining the observed stylized facts, these assumptions are necessary. We will show Bayesian models with common priors can deliver similar findings with the help of higher order expectations.

The structure of this paper is as follows. In the next section we connect disagreement increasing public announcements to higher-order expectations and asset pricing. For expositional purposes, in that section we abstract from the informational role of prices and we use a simple dynamic structure. In section 3, we present the full model with learning from prices and more reasonable dynamics and we discuss the results. In section 4 we confront our findings with existing empirical results. Finally we conclude.

\section{Structures with increasing disagreement and higher-order expec- tations}

In this section, we characterize information structures with increasing disagreement and argue that such information structures naturally arise in a financial market context where early traders buy for resale and consequently trade on their expectation of the expectation of future traders. In the first part, we show the intuition behind our model with the help of a simple example, and we discuss the crucial property of higher-order expectations from the perspective of this paper. We constrain ourselves to the normal case in that subsection. In the second part, we analyze the interesting cases of disagreement increasing public announcements in a general environment and we show why higher-order expectations provide a convenient way to have increasing disagreement in an asset-pricing model.

\subsection{Higher-order expectations and public announcements: an example}

Let us present a modified version of Keynes' beauty contest example.

Example 1 (Restaurant-contest) A newly opened restaurant is assessed. The quality of the restaurant, $\theta$, depends on two factors, the quality of food, $\theta_{f}$, and the quality of the environment, $\theta_{e}$ where $\theta=\theta_{f}+\theta_{\varepsilon}$. There is a one-member-jury, who cannot visit the restaurant, she only makes her judgement by tasting a sample of the food, $x=\theta_{f}+\varepsilon$. There is also a group of forecasters, $j=1,2 \ldots J$, who guess the judgement of the jury and the one whose guess is closest to the actual judgement gets a prize. These forecasters have not had the possibility to taste the food yet but each of them visited the building-site once, each in a different phase of the construction. So they have some information on the environment: $x_{j}=\theta_{e}+\varepsilon_{j}$ We will be interested in the change of dispersion of forecasts before and after the disclosure of a public piece of information on the quality of the restaurant, $y=\theta+\eta$, a review published in the local paper. We assume that the variables $\theta_{f}, \theta_{e}, \varepsilon, \varepsilon_{j}, \eta$ are all have zero mean and they are independent and normally distributed. Hence, the judgment of the jury will be a 
linear function of her signals. If the review is not published, it will be $E(\theta \mid x)=b_{\theta} x$ and it will be $E(\theta \mid x, y)=b_{\theta}^{\prime} x+c_{\theta}^{\prime} y$, if it is published, where $b_{\theta}, c_{\theta}$ and $c_{\theta}^{\prime}$ are functions of variances and covariances of the random variables. Without the review forecasters will not have any information on the quality of the food, hence they will not know anything on the signal of the jury. Hence, forecasts will be identical across forecasters in a trivial manner:

$$
E_{j}\left(E(\theta \mid x) \mid x_{j}\right)=E_{j}\left(b_{\theta} x \mid x_{j}\right)=b_{\theta} E_{j}\left(\theta_{f}+\varepsilon \mid \theta_{e}+\varepsilon_{j}\right)=0 .
$$

However, if the review is published, there will be dispersion in the opinion of the forecasters as their forecasts will be sensitive to their private signal

$$
\begin{aligned}
E_{j}\left(E(\theta \mid x, y) \mid x_{j}, y\right) & =E_{j}\left(b_{\theta}^{\prime} x+c_{\theta}^{\prime} y \mid x_{j}, y\right)=b_{\theta}^{\prime} E_{j}\left(\theta_{f}+\varepsilon \mid \theta_{e}+\varepsilon_{j}, \theta_{e}+\theta_{f}+\eta\right)+c_{\theta}^{\prime} y= \\
& =b_{\theta}^{\prime}\left(b_{x}^{\prime} x_{j}+b_{y} y\right)+c_{\theta}^{\prime} y .
\end{aligned}
$$

The critical points in our example are that the connection between the information set of the jury and that of the forecasters is weak (zero) and the public signal is correlated to both. Although our forecasters have relevant information on the quality of the restaurant, this is not what they have to guess. They do not have information on the jury's information. Hence, their guess is simply the a priori mean of the information of the jury. However, the review connects their information to the information of the jury. After observing the public information, forecasters can use their private information. Because their private information differs, so do their forecasts. In particular, those who think that the environment is very nice - contributing a larger part of a positive review to the environment factor than they should - will underestimate the quality of the food and the assessment of the jury ${ }^{3}$. Those with unfavorable information on the environment will do just the opposite. So the variance of the forecasts increases due to the review.

In our model, instead of a one-member jury assessing the restaurant there will be a group of traders who will asses the quality of a risky asset. Their assessment will be reflected in the price of the last period. Early traders - in the role of the forecasters - will be interested in this price, because they will be forced to liquidate their positions early, at this price. But for now, let us generalize our example to see how higher than second-order expectations behave and what are the precise conditions for the correlation structure for disagreement to increase after an announcement. So, we will assume that there are many groups of forecasters, $t=1 \ldots T$, and these groups are ordered, where now the $T$ th group of forecasters will play the role of the jury. Each of them has to forecast the next group's average forecast in the line. For example, a trader $j$ 's forecast with and without the announcement in group $T-2$ will be her third-order expectation

$$
\begin{aligned}
E^{3}\left(\theta \mid x_{T-2, j}, y\right) & =E_{j}\left(\bar{E}\left(\bar{E}\left(\theta \mid x_{T, j}, y\right) \mid x_{T-1, j}, y\right) \mid x_{T-2, j}, y\right) \text { and } \\
E^{3}\left(\theta \mid x_{T-2, j}\right) & =E_{j}\left(\bar{E}\left(\bar{E}\left(\theta \mid x_{T, j}\right) \mid x_{T-1, j}\right) \mid x_{T-2, j}\right),
\end{aligned}
$$

\footnotetext{
${ }^{3}$ This adverse relationship between the agents' information on the fundamental and their guess on other's guess on the fundamental is the focus in the companion paper of Kondor (2004). In this paper we focus on increasing dispersion only.
} 
where $\bar{E}(\cdot)$ is the average of the forecasts in the group, $x_{t, j}$ is the private signal of agent $j$ in group $t$ and $y$ is the public announcement. We will still assume normally distributed signals, but we allow for a more general covariance structure. Let us assume that covariances between any two of our random variables are positive. For the sake of simplicity, we will assume a symmetric structure, where the variance of every private signal is $\sigma_{x}^{2}$, the covariance between any private signal and the fundamental value or the public signal are $\sigma_{\theta, x}$ and $\sigma_{y, x}$ respectively and all variables have zero mean. Furthermore, the covariance between two private signals in the same group is $\sigma_{x, x}$, while the covariance between private signals in different groups is $\sigma_{x, x^{\prime}}$. The only assumption we make on the relative sizes of covariance and variance terms is that the private signal $x_{t}^{i}$ and the public signal, $y$, are positive $\operatorname{signals}^{4}$ on $\theta$ for agent $i$ in period $t$ in the presence of the public signal i.e. a higher $x_{t}^{i}$ or a higher $y$ will rise the fundamental expectation of agent $i$ :

$$
\begin{aligned}
& b_{\theta}^{\prime}=\frac{\partial E\left(\theta \mid x_{t}^{i}, y\right)}{\partial x_{t}^{i}}=\frac{\sigma_{\theta, . x} \sigma_{y}^{2}-\sigma_{x, y} \sigma_{\theta, y}}{\sigma_{x}^{2} \sigma_{y}^{2}-\sigma_{x, y}^{2}}>0 \\
& c_{\theta}^{\prime}=\frac{\partial E\left(\theta \mid x_{t}^{i}, y\right)}{\partial y}=\frac{-\sigma_{x, \theta} \sigma_{x, y}+\sigma_{\theta, y} \sigma_{x}^{2}}{\sigma_{x}^{2} \sigma_{y}^{2}-\sigma_{x, y}^{2}}>0 .
\end{aligned}
$$

It is easy to check that $b_{\theta}^{\prime}, c_{\theta}^{\prime}>0$ implies that the private signal is a positive signal on $\theta$ in the no-announcement case as well - i.e. $b_{\theta}=\frac{\partial E\left(\theta \mid x_{t}^{i}\right)}{\partial x_{t}^{i}}=\frac{\sigma_{\theta, x}}{\sigma_{x}^{2}}>0$ - and that $\left|b_{\theta}\right|>\left|b_{\theta}^{\prime}\right|$. This condition implies that public announcements will never increase disagreement over the fundamental value i.e. $b_{\theta}^{\prime}, c_{\theta}^{\prime}>0$ implies $b_{\theta}>b_{\theta}^{\prime}$. In contrast to that, the next proposition shows that all higher-order expectations become more sensitive to private signal after the announcement, if private information sets across periods are not too strongly correlated. Hence, dispersion within groups increase due the announcement. The intuition again is that with higher-order expectations agents' guess on the fundamental value does not matter; the critical point is what each agent knows about another agent's signal.

Proposition 1 If $x_{t}^{i}$ and $y$ are positive signals i.e. $b_{\theta}, c_{\theta}>0$ and

$$
\frac{b_{\theta} \rho_{x, y}^{2}}{\left(b_{\theta}+\rho_{\theta, x}\left(1-\rho_{x, y}^{2}\right)\right)}>\rho_{x^{\prime}, x},
$$

where $\rho_{v_{1}, v_{2}}$ is the correlation between $v_{1}$ and $v_{2}$ then

$$
\left|\frac{\partial E\left(\bar{E}^{k}(\theta) \mid x_{j t}\right)}{\partial x_{t}}\right|<\left|\frac{\partial E\left(\bar{E}^{k}(\theta) \mid x_{j t}, y\right)}{\partial x_{t}}\right|
$$

holds for all $k \geq 1$.

\footnotetext{
${ }^{4}$ In a more general set-up, this assumption would correspond to the asumption of positive affiliation of $\left(\theta, x_{t}^{i}, y\right)$ for all $t$ and $i$. (Our assumption is weaker, but works for the normal case only.) In terms of affiliation, the interesting property of our information structure is that although $\left(\theta, x_{t}^{i}, y\right)$ is affiliated, $\left(x_{t^{\prime}}^{i}, x_{t}^{i}, y\right)$ - the system of the public signal and the private signals of two agents from two different periods - is not. This is why belief swap is possible in higher-order expectations. For a general discussion of similar information structures in an auction context see Jewitt (2004).
} 
Proof. Let us introduce $b_{x}^{\prime}$, and $b_{x}$ for the coefficients of private signals in traders' conditional expectations on other traders private signals in other periods:

$$
\begin{aligned}
& b_{x}^{\prime}=\frac{\sigma_{x, x^{\prime}} \sigma_{y}^{2}-\sigma_{x, y}^{2}}{\sigma_{x}^{2} \sigma_{y}^{2}-\sigma_{x, y}^{2}}=\frac{\partial E\left(x_{u}^{j} \mid x_{t}^{i}, y\right)}{\partial x_{t}^{i}} \text { for all } u \neq t, \\
& b_{x}=\frac{\sigma_{x, x^{\prime}}}{\sigma_{x}^{2}}=\frac{\partial E\left(x_{u}^{j} \mid x_{t}^{i}\right)}{\partial x_{t}^{i}} \text { for all } u \neq t .
\end{aligned}
$$

Note that

$$
\left|\frac{\partial E\left(\bar{E}^{k}(\theta) \mid x_{j t}\right)}{\partial x_{t}}\right|=\left|b_{x}^{k} b_{\theta}\right| \text { and }\left|\frac{\partial E\left(\bar{E}^{k}(\theta) \mid x_{j t}, y\right)}{\partial x_{t}}\right|=\left|b_{x}^{\prime k} b_{\theta}^{\prime}\right|
$$

As $b_{\theta}>b_{\theta}^{\prime}>0,\left|b_{x}\right| b_{\theta}<\left|b_{x}^{\prime}\right| b_{\theta}^{\prime}$ implies $\left|b_{x}\right|<\left|b_{x}^{\prime}\right|$. Therefore, $\left|b_{x}\right| b_{\theta}<\left|b_{x}^{\prime}\right| b_{\theta}^{\prime}$ implies (2). The condition in the proposition comes directly - after substitution and straightforward manipulation from $\left|b_{x}^{n}\right| b_{\theta}^{n}<\left|b_{x}\right| b_{\theta}$.

\section{Asset pricing and information structures with increasing disagree- ment}

Before we make the connection to financial applications, let us explore the interesting cases of increasing disagreement due to public information in general. We consider agents indexed by $j$ who have to form an opinion on an issue. First, let us assume that there are only two agents $j=A, B$. We assume a two-step process. They start with the same priors, but in the first step each receives some private information, $I_{j}$. This private information is responsible for the initial disagreement. We ask them for their opinion, $o_{j}$ at that point. Then a public piece of information, $y$ is also revealed. We ask their opinion, $o_{j}^{\prime}$, again. We are interested in the change in agents' opinion due to the public information release. The issue will be represented by the random variable $\phi$. Their initial opinion will be their expectation of $\phi$ given their private information sets:

$$
o_{j}=E\left(\phi \mid I_{j}\right) \quad j=A, B
$$

Their final opinion is

$$
o_{j}^{\prime}=E\left(\phi \mid I_{j}, y\right) \quad j=A, B
$$

With two agents, disagreement can increase after public announcements in four different ways. It is possible that the opinions of both agents improve, but the optimist's improve more. The same is possible to the opposite direction. It is also possible that each agent gets even more convinced of his or her original opinion, and finally, disagreement can also increase when the optimist becomes the pessimist and vice versa and the change is large enough. The first two cases are rather qualitative than quantitative phenomena, and they occur quite naturally for certain announcements in most information structures, so we will not deal with them in this discussion. We will focus on the more surprising last two possibilities when the public information moves opinions very differently across 
agents: polarization and belief swap.

Definition 1 There is increasing disagreement for $A, B$ and information y if $\left|o_{A}^{\prime}-o_{B}^{\prime}\right|>\left|o_{A}-o_{B}\right|$, there is polarization for $A, B$ and $y$ if $o_{A}>o_{B}$ implies

$$
o_{A}^{\prime}>o_{A} \text { and } o_{B}^{\prime} \leq o_{B}
$$

There is belief swap if $o_{A}>o_{B}$ implies $o_{A}^{\prime}<o_{B}^{\prime}$.

It should be clear that polarization implies increasing disagreement, and belief swap can happen with and without increasing disagreement. In our full model - similar to Example 1 - the public signal will cause belief swaps together with increasing disagreement for any two agents with different private signals and for any announcement.

To illustrate our concepts, we present a second example. It also demonstrates that there are environments with increasing disagreement where higher-order expectations do not play a role. There are two possible public announcements and one of them causes belief swap while the other causes polarization and both of them result in increasing disagreement.

Example 2 Suppose that investors are waiting for the opinion of a financial analyst on a particular firm. ${ }^{5}$ The firm can be valuable $(V)$ or worthless $(W)$. However, the analyst is also one of two types. It is either an enemy (E), who always gives bad advice, - possibly because some conflicts of interest as a consequence of being a branch of an investment bank - or a friend (F) who always gives good advice. The prior distribution about these states is the same for all investors:

\begin{tabular}{|l||l|l|}
\hline & $V$ & $W$ \\
\hline \hline$F$ & $p$ & $\frac{1-2 p}{2}$ \\
\hline$E$ & $\frac{1-2 p}{2}$ & $p$ \\
\hline
\end{tabular}

where $\frac{1}{4}<p<\frac{1}{2}$. So the a priori chance of the firm being valuable is $\frac{1}{2}$, but-for some reason - there is some correlation between the type of the analyst and the type of the firm: valuable firms tend to go to friend analysts and vice-versa. There are two steps of information arrivals. First each investor receives a noisy information $i$ about the type of the analyst. Let us assume that $i_{A}=F$ and $i_{B}=E$. This signal is true with probability $1>q>\frac{1}{2}$. After this signal, we ask investors about their probability assessment of the firm being a valuable investment possibility: $o_{A}, o_{B}$. In the second step the analyst announces its report which is either that the firm is valuable, $y=V$, or that the firm is worthless, $y=W$. We assume that the agency knows the value of the firm for certain. We ask the investors

\footnotetext{
${ }^{5}$ We thank Enrico Sette for this interpretation of our example.
} 
again: $o_{A}^{\prime}, o_{B}^{\prime}$. It is easy to check by Bayes' Rule that

\begin{tabular}{|l||c|c|c|}
\hline & before announcement & after announcement $V$ & after announcement $W$ \\
\hline \hline investor $A: o_{A}, o_{A}^{\prime}$ & $4 q p+1-2 p-q$ & $q$ & $1-q$ \\
\hline investor $B: o_{B}, o_{B}^{\prime}$ & $2 p-4 q p+q$ & $1-q$ & $q$ \\
\hline difference: $o_{A}^{(\prime)}-o_{B}^{(\prime)}$ & $(4 p-1)(2 q-1)>0$ & $2 q-1$ & $1-2 q$ \\
\hline
\end{tabular}

Hence, we have polarization, if $y=V$, belief swap if $y=W$, and disagreement increase in both cases.

Note, that the restaurant-contest example is analogous in many respects to this one. Although in the first example the pay-off relevant state for the forecasters is the judgement of the jury, while here it is the quality of the firm. And although their private information is about the environment of the restaurant while here it is about the type of the analyst, the critical points remain the same. In both cases the private signal itself does not give too much information about the relevant variable: the public information, i.e. the review about the restaurant or the announcement of the analyst, makes the difference. Furthermore, conditionally on the pay-off relevant state, the public information is not independent of the private information: the announcement of the analyst means something different for the two agents and the published review means something different for the forecasters. However, the two examples do differ in a very important way. In this second example, we need very specific public announcements. We have to assume that the announcer is either an enemy or a friend and the decision makers have different private information on the type of the announcer. In the rest of this section, we will show that these strong assumptions are not specific to our example; we need similar ones to generate increasing disagreement in models without higher-order expectations. It would be very hard to find an analogue to this set-up in financial markets. In contrast, in the first example higher-order expectations help us to have increasing disagreement without having such strong assumptions on the type of the announcer. We only need some agents who enter earlier and form expectations on the opinion of late-entrants, not too correlated private information sets across groups and public announcements which is correlated with all private information sets. We will argue later that these are very plausible assumptions for financial markets. In effect, we substitute assumptions on the information structure for assumptions on the time of entry and exit of traders. This is the main idea of the paper.

From now on, let us assume that the private piece of information of each individual $i$, consists of a single private signal $x_{i}$, and there are infinitely many agents. The distribution of the pay-off relevant state $\phi$, the vector of public signals $y$ and private signals $x_{i}$ is characterized by the density function $f\left(\phi, x_{i}, y\right)$. Hence, for the sake of simplicity, we assume that conditional on $\phi$ and $y$, private signals are drawn independently from the same distribution. Furthermore, we assume that the marginal density function of any subset of our random variables exists and is also differentiable with respect to any of our variables and all of these densities have a full support on a given closed state space $S \subseteq R^{2+n_{y}}$ 
where $n_{y}$ is the dimension of $y^{6}$. Let us also assume that $\frac{\partial^{2} \ln f\left(\phi \mid x_{i}\right)}{\partial \phi \partial x_{i}}>0$ so from Milgrom $(1981)^{7}$ $E\left(\phi \mid x_{i}\right)$ is increasing in $x_{i}$. It is then not too hard to give simple sufficient conditions for belief swap and polarization.

\section{Proposition 2 1. If}

$$
\begin{aligned}
& \frac{\partial \ln f\left(y \mid \phi, x^{\prime \prime}\right)}{\partial \phi}>0 \text { but } \\
& \frac{\partial \ln f\left(y \mid \phi, x^{\prime}\right)}{\partial \phi}<0 \text { for all } \phi
\end{aligned}
$$

then there is polarization for the given $x^{\prime \prime}>x^{\prime}$ and $y$., where $x^{\prime}, x^{\prime \prime}, y, \phi \in S$.

2. If

$$
-\frac{\partial^{2} \ln f(y \mid \phi, x)}{\partial \phi \partial x}<\frac{\partial^{2} \ln f(\phi \mid x)}{\partial \phi \partial x}
$$

for the given $y$ and all $\phi, x \in S$ then there is belief swap for $y$ and for all $x$.

Proof. From Milgrom (1981), if $\frac{\partial \ln g_{1}(\phi)}{\partial \phi}>\frac{\partial \ln g_{2}(\phi)}{\partial \phi}$ where $g_{1}(\cdot), g_{2}(\cdot)$ are two distribution functions, then $E_{1}(\phi)>E_{2}(\phi)$ holds. Hence, for polarization, it is sufficient if

$$
\frac{\partial \ln f\left(\phi \mid x^{\prime \prime}, y\right)}{\partial \phi}>\frac{\partial \ln f\left(\phi \mid x^{\prime \prime}\right)}{\partial \phi} \text { and } \frac{\partial \ln f\left(\phi \mid x^{\prime}, y\right)}{\partial \phi}<\frac{\partial \ln f\left(\phi \mid x^{\prime}\right)}{\partial \phi} .
$$

Similarly, for belief swap it is sufficient if

$$
\frac{\partial^{2} \ln f(\phi \mid x, y)}{\partial \phi \partial x}<0<\frac{\partial^{2} \ln f(\phi \mid x)}{\partial \phi \partial x} .
$$

But

$$
\begin{aligned}
\ln f(\phi \mid x, y) & =\ln \frac{f(\phi, x, y)}{f(x, y)}=\ln \frac{f(y \mid \phi, x) f(\phi \mid x)}{f(y \mid x)}= \\
& =\ln f(y \mid \phi, x)+\ln f(\phi \mid x)-\ln f(y \mid x)
\end{aligned}
$$

which - by substituting back into the inequalities above - gives all the results.

Our conditions are in line with the intuition provided by our second example. There is polarization if the public announcement is good news if the agent knows $x^{\prime \prime}$ but bad news if she knows $x^{\prime} .^{8}$

\footnotetext{
${ }^{6}$ The generalization of any of the results below for the discrete case would be a straightforward exercise.

${ }^{7}$ It is that $f\left(\theta \mid x_{i}\right)$ is $\log$-supermodular in $\theta$ and $x_{i}$ or that the log-likelihood ratio property is satisfied: $\frac{f\left(\theta^{\prime \prime} \mid x^{\prime \prime}\right)}{f\left(\theta^{\prime} \mid x^{\prime \prime}\right)}>$ $\frac{f\left(\theta^{\prime \prime} \mid x^{\prime}\right)}{f\left(\theta^{\prime} \mid x^{\prime}\right)}$ for all $\theta^{\prime \prime}>\theta^{\prime}$ and $x^{\prime \prime}>x^{\prime}$. As it is shown in Milgrom (1981), this is sufficient condition for second order stochastic dominance and consequently is sufficient for $E(\theta \mid x)$ to be increasing in $x$.

${ }^{8}$ Milgrom (1981) uses the definition that news $z^{\prime \prime}$ is more favourable than $z$ iff

$$
\begin{aligned}
\frac{f\left(z^{\prime \prime} \mid \theta^{\prime \prime}\right)}{f\left(z^{\prime \prime} \mid \theta^{\prime}\right)} & >\frac{f\left(z^{\prime} \mid \theta^{\prime \prime}\right)}{f\left(z^{\prime} \mid \theta^{\prime}\right)} \text { or } \\
\frac{\partial \ln f\left(z^{\prime \prime} \mid \theta\right)}{\partial \theta} & >\frac{\partial \ln f\left(z^{\prime} \mid \theta\right)}{\partial \theta} .
\end{aligned}
$$
}


Furthermore, there is belief swap if the larger the $x$, the worse news is $y$ and if this effect is strong relative to the effect of the private signal on the probability of $\phi$ in the absence of public information.

Although we cannot find similarly simple conditions for disagreement increase in general, proposition 2 helps us to connect higher-order expectations, structures with disagreement increase and asset-pricing in the simplest CARA-Normal world. So let us turn to our financial application and use the simplest possible asset-pricing model with asymmetric information. Now, our agents will be traders in a market of a risky asset. They will trade for one period only. Instead of the effect of the public announcement on their opinion, we will be interested in its effect on their demand for the asset. Each agent has a constant absolute risk-aversion utility $U_{j}\left(W_{j}\right)=-e^{-a_{j} W_{j}}$, where $W_{j}$ is their end of period wealth, and $a_{j}$ is the measure of risk-aversion. Here, the issue, $\phi$, which they form an opinion on, will be the value of the asset at the end of the period. There is a random supply of the asset, so prices are not fully revealing. A trader $j$ will receive a private signal $x_{j}$ and possibly the public information $y$. We assume that all random variables are jointly normal and that traders are symmetric i.e. the distribution of $x_{i}$ conditional on the other variables are the same across all $i$.

Then the demand of trader $j$ in period $t$ will be

$$
d_{j}=\frac{E\left(\phi \mid x_{j}, p\right)-p}{a_{j} \operatorname{var}\left(\phi \mid x_{j}, p\right)}
$$

if there is no announcement and

$$
d_{j}^{\prime}=\frac{E\left(\phi \mid x_{j}, p, y\right)-p}{\operatorname{avar}\left(\phi \mid x_{j}, y, p\right)}
$$

if there is announcement. These expressions show the familiar relationship that the larger the disagreement - the numerator - or the larger the precision - smaller the denominator - the larger is the position. As precision increases, by the arrival of the announcement, increasing disagreement leads to larger positions. Similarly, it is clear that the speculative trading volume will be larger ${ }^{9}$, if each trader trades more aggressively due to the announcement i.e. trading intensity increases:

$$
\left|\frac{\partial d_{j}}{\partial x_{j}}\right|<\left|\frac{\partial d_{j}^{\prime}}{\partial x_{j}}\right| .
$$

Because of linearity of demand functions, this will be satisfied either for all agents or for none of them. Furthermore, - because variance is independent of the realized signal - this is exactly the condition for increasing disagreement between any two of the agents in the same group.

For the rest of the section, let us assume that traders do not learn from prices. There will be learning from prices in the full model, here we abstract it away only to strengthen our intuition. Now,

In that sense, our sufficient condition for polarization can be interpreted that $z$ is more favourable when our agent knows $x^{\prime \prime}$ than if she knows $x^{\prime}$.

${ }^{9}$ We will be more precise on the definition of trading volume and its relation to speculative trades and risk-sharing trades later in the text. The present discussion is intended to remain on an intuitive level. 
we can make two observations. The first one is, that with normally distributed variables

$$
\begin{aligned}
\frac{\partial d_{j}}{\partial x_{j}} & =\frac{1}{a_{j}} \frac{\frac{\partial E\left(\phi \mid x_{j}\right)}{\partial x_{j}}}{\operatorname{var}\left(\phi \mid x_{j t}\right)}=\frac{1}{a_{j}} \frac{\partial^{2} \ln f\left(\phi \mid x_{j}\right)}{\partial \phi \partial x_{j}} \\
\frac{\partial d_{j}^{\prime}}{\partial x_{j}} & =\frac{1}{a_{j}} \frac{\frac{\partial E\left(\phi \mid x_{j}, y\right)}{\partial x_{j}}}{\operatorname{var}\left(\phi \mid x_{j}, y\right)}=\frac{1}{a_{j t}} \frac{\partial^{2} \ln f\left(\phi \mid x_{j}, y\right)}{\partial \phi \partial x_{j}} .
\end{aligned}
$$

As we know that

$$
\ln f\left(\phi \mid x_{j}, y\right)=\ln f\left(y \mid \phi, x_{j}\right)+\ln f\left(\phi \mid x_{j}, y\right)-\ln f\left(y \mid x_{j}\right),
$$

we also know that

$$
\frac{\partial d_{j}^{\prime}}{\partial x_{j}}=\frac{\partial d_{j}}{\partial x_{j}}+\frac{1}{a_{j}} \frac{\partial^{2} \ln f\left(y \mid \phi, x_{j}\right)}{\partial \phi \partial x_{j}}=\frac{\partial d_{j}}{\partial x_{j}}-\frac{\frac{\partial E\left(y \mid \phi, x_{j}\right)}{\partial x_{j}} \frac{\partial E\left(y \mid \phi, x_{j}\right)}{\partial \phi}}{a_{j t} \operatorname{var}\left(y \mid \phi, x_{j}\right)}
$$

With the logic of proposition 2, this equation gives necessary and sufficient conditions for increasing disagreement with belief swap in the following proposition.

Proposition 3 When prices are not informative, traders will reverse their bets due to the announcement, if and only if

$$
\frac{\frac{\partial E\left(y \mid \phi, x_{j}\right)}{\partial x} \frac{\partial E(y \mid \phi, x)}{\partial \phi}}{a_{j} \operatorname{var}\left(y \mid \phi, x_{j}\right)}>\frac{\partial d_{j}}{\partial x_{j}}=\frac{1}{a_{j}} \frac{\frac{\partial E\left(\phi \mid x_{j}\right)}{\partial x_{j}}}{\operatorname{var}\left(\phi \mid x_{j t}\right)}
$$

and they also trade more aggressively if and only if

$$
\frac{\frac{\partial E\left(y \mid \phi, x_{j}\right)}{\partial x_{j}} \frac{\partial E\left(y \mid \phi, x_{j t}\right)}{\partial \phi_{t}}}{a_{j} \operatorname{var}\left(y \mid \phi, x_{j}\right)}>2 \frac{\partial d_{j}}{\partial x_{j}}=\frac{2}{a_{j}} \frac{\frac{\partial E\left(\phi \mid x_{j}\right)}{\partial x_{j}}}{\operatorname{var}\left(\phi \mid x_{j t}\right)} .
$$

The last expression shows, why a structure with higher-order expectations with early-traders and latecomers, can result in increasing trading activity after an announcement as opposed to other models. Conditions (3) and (4) will hold if $\frac{\partial E\left(y \mid \phi, x_{j}\right)}{\partial x_{j}} \frac{\partial E\left(y \mid \phi_{t}, x_{j t}\right)}{\partial \phi_{t}}$ is large. It means that $\phi$ and $x$ contain complementary information on the public signal $y$. In a standard model without higher-order expectations every agent is interested in the fundamental value, $\theta$, so $\phi=\theta$. Then finding an information structure where private signals contain additional information to the true value on the public signal is very demanding ${ }^{10}$. For example, with the usual assumption that the announcement is a noisy version of the true value, $y=\theta+\eta$, where $\eta$ is uncorrelated to $x_{j}$, the private signal cannot possibly improve the estimation of the public signal beyond the true value, i.e. $\frac{\partial E\left(y \mid \phi, x_{j}\right)}{\partial x_{T}}=\frac{\partial E(y \mid \theta, x)}{\partial x_{T}}=0$. In contrast, we may assume that there are early trader indexed by $j$ and latecomers indexed by $i$. Early traders arrive

\footnotetext{
${ }^{10}$ However, an information structure, where $\phi=\theta$ and still the private signal improves the estimate $E\left(y \mid \theta, x_{j}\right)$, is certainly possible. For example, in Verrecchia (1997) agents have private information on the error in the public announcement, so $\frac{\partial E\left(y \mid \theta, x_{j}\right)}{\partial x_{j}} \neq 0$. Still, given that for more aggressive trading, we need $\frac{\partial E\left(y \mid \theta, x_{j}\right)}{\partial x_{j}}$ to be large enough compared to $\frac{\partial E\left(\theta \mid x_{j}\right)}{\partial x_{j}}$, we believe that this argument is much more demanding in terms of informational assumptions than our own.
} 
first, they trade then they sell their holdings to latecomers who trade and liquidate for the true value $\theta$. Thus an early trader is interested in the second period price, not the true value: $\phi_{j}=p_{2}=f\left(x_{i}\right)$, which will be a function of the information of last period traders only. Hence, on the left hand side of conditions (3) and (4) we will have

$$
\frac{\frac{\partial E\left(y \mid p_{2}, x_{j}\right)}{\partial x_{j}} \frac{\partial E\left(y \mid p_{2}, x_{j t}\right)}{\partial p_{2}}}{a_{j} \operatorname{var}\left(y \mid \phi, x_{j}\right)}=\frac{\frac{\partial E\left(y \mid f\left(x_{i}\right), x_{j}\right)}{\partial x_{j}} \frac{\partial E\left(y \mid f\left(x_{i}\right)_{t}, x_{j t}\right)}{\partial f\left(x_{i}\right)}}{a_{j} \operatorname{var}\left(y \mid f\left(x_{i}\right), x_{j}\right)}
$$

Conditions (3) and (4) will hold if these expressions are large enough. It means that $x_{j}$ should give additional information on $y$ apart from the information contained in $p_{2}=f\left(x_{i}\right)$ should give additional information on $y$ apart from that contained in $x_{j}$. This will be possible if $x_{j}$ and $x_{i}$ are not too related to each other, but both of them are related to $y$. This is exactly the case both in Example 1 and in our full model in the next section.

\section{The model}

\subsection{The set-up}

We modify a standard, dynamic, CARA-Normal, rational expectations model with differential information (e.g. He and Wang, 1995, Brown and Jennings, 1989). As in any similar model since Grossman-Stiglitz (1980), preferences of our traders are given by $U_{i}\left(W_{i}\right)=-e^{-a W_{i}}$, where $W_{i}$ is monetary wealth at the time of the exit, $a$ is the absolute risk-aversion parameter and in each period traders submit demand curves to an auctioneer to buy up the random supply of assets: $u_{t} \sim N\left(0, \frac{1}{\delta_{t}^{2}}\right)$. Traders base their portfolio decision on the private signal which they receive at the moment of their entry and all available public signals i.e. past and present prices and public announcements. They update their beliefs by Bayes' Rule. Prices, $p_{t}$, are determined by market clearing.

However, as a non-standard assumption, we will have two groups of traders - with continuum traders in each group - and $2+1$ periods $(t=0,1,2)$. Traders in the first group trade among themselves in periods 0 and 1 and sell all of their remaining assets in period 2. Traders in the second group trade among each other in period 2 and liquidate for the uncertain value of $\theta$ at the end of the game. For expositional purposes only, let us interpret our model in terms of a 24-hour day in the USD/GBP market. However, the reader should keep in mind that our set up would fit to any market, where traders focus on the resale value of their assets instead of their fundamental value. With the FX interpretation, the first group represents traders based in London, while the second group is based in New York. Period 0 and 1 are daylight periods in London, so Londoners trade among themselves twice, and then they go to sleep, so they sell all their holdings to New Yorkers. They do not hold positions overnight ${ }^{11}$. Period 2 is daylight in New York, so New Yorkers trade among themselves and get $\theta$ in the evening. We assume that if there is a public announcement, $y$, then it will be released at the beginning of period 1 . Hence, we will focus on the differences in trading patterns of Londoners

\footnotetext{
${ }^{11}$ Although we use the interpretation of a 24-hour FX market only for expositional reasons, it happens to be a stylized fact among FX dealers that they do not hold positions overnight (see Lyons, 2001).
} 
(early traders) with and without an announcement.

The driving force of our model lies in the information structure. We assume that the fundamental value of the asset - the exchange rate in this interpretation- is given by

$$
\theta=\theta_{s}+\theta_{k}+\theta_{w}
$$

where $\theta_{s}, \theta_{k}, \theta_{w}$ are the US factor, the UK factor and the world factor respectively. We assume that the private signal that Londoners receive contains noisy information on the UK factor and the world factor, but does not contain information on the US factor: $x_{i}=\theta_{k}+\theta_{w}+\varepsilon_{i}$, while the private signals of New Yorkers contain information on the US factor and the world factor, but not on the UK factor: $z_{j}=\theta_{s}+\theta_{w}+\varepsilon_{j}$. Hence, the world factor simply represents the common element in the information set of agents in different groups, while the US factor and the UK factor represent group-specific information. The public signal contains information on fundamental value: $y=\theta+\eta$. We assume that all factors and noise terms are i.i.d. and normally distributed:

$$
\theta_{k}, \theta_{s} \sim N\left(0, \frac{1}{\kappa}\right), \theta_{w} \sim N\left(0, \frac{1}{\omega}\right), \varepsilon_{i}, \varepsilon_{j} \sim N\left(0, \frac{1}{\alpha}\right), \eta \sim N\left(0, \frac{1}{\beta}\right)
$$

As we will see, our model gives new insights when $\omega$ is large i.e. when the common component in the information structure is relatively small. Intuitively, high- $\omega$ case describes a situation, where agents in different groups has information of different parts of the big picture, while the public signal is about the big picture. We believe that this is a fairly typical case. After all, while the public signal is mostly about aggregates, like macroeconomic indicators on the FX market or earning announcements in the stock market, private information is usually about some specific factors of the asset value. For example, in the case of a dealer in the foreign exchange, the typical piece of private information would be the change of order flow of her particular consumer base (see Lyons, 2001), or in the case of an insider at the stock market it would be some information about the outcome of a particular project or a transaction. As another dealer has a different consumer base - possibly with different characteristics - and an other insider might have information about a different transaction, these pieces of private information are arguably not very correlated across agents in different groups.

It is instructive to compare our structure to information in other rational expectations models. The following table presents the structure of some of the most prominent models in the literature.

\begin{tabular}{|l||l|l|l|}
\hline model & private s. & public s. & liquidation v. \\
\hline \hline Brown-Jennings(1989) Kim-Verrecchia(1991,1994) & $\theta+\varepsilon_{i}$ & $\theta+\eta$ & $\theta$ \\
\hline He and Wang(1995) & $\theta+\varepsilon_{i}$ & $\theta+\eta$ & $\theta+\xi$ \\
\hline
\end{tabular}

Note, that all these models, together with the majority of asymmetric information models in finance 12 , use a one-factor framework. The problem is that the assumption that all signals are noisy versions of the fundamental, imposes a very rigid structure on information sets. Namely, all covariances

\footnotetext{
${ }^{12}$ Foster and Viswanathan (1996) is a notable exception. For a detailed review of the literature, see Brunnermeier (2001).
} 
between any two of the random variables equals the variance of the fundamental value: $\operatorname{cov}\left(x_{i}, x_{j}\right)=$ $\operatorname{cov}\left(x_{i}, y\right)=\operatorname{cov}\left(x_{i}, \theta\right)=\operatorname{var}(\theta)$. Our structure represents a partial relaxation of this assumption. We allow for weaker correlation between private signals across groups. We presented the structure with the help of a specific story about the FX traders just for exponential purposes. We believe that our model applies to any financial markets, where traders cannot be sure to be able to hold their positions until it is optimal and where those to whom they will sell, do not necessarily have a very similar information set to theirs.

In the one-factor structure, disagreement never increases due to an announcement. In particular, in the simplest Brown and Jennings model, there is no effect of public announcement at all: increased precision and decreased disagreement exactly cancels out. In the Kim-Verrecchia (1991) case there is some volume due to different precision of signals of different traders, but the effect is small and always proportional to the price change. In He and Wang (1995), there is an additional random factor in the liquidation value, $\xi$, which is not included in the union of the traders' information set. This induces traders to follow a more complex dynamic strategy over time, which allows traders to bet in advance on the price effect of the public announcement. Hence, they will build up positions before the announcement and liquidate these positions when the announcement is released. This effect works only with expected announcements. Our model will deliver this bet-in-advance effect as well, but we will have an additional effect coming from increased disagreement, which will work even if the announcement is unexpected.

Our model nests the information structures of these standard models. The next table summarizes the connections.

\begin{tabular}{|l||l|l|l|l|l|}
\hline our parameter & private & public & fundamental & $p_{2}(\cdot)$ & model \\
\hline \hline$\kappa, \delta_{2} \rightarrow \infty$ & $\theta_{w}+\varepsilon_{i}$ & $\theta_{w}+\eta$ & $\theta_{w}$ & $\approx \theta_{w}$ & $\begin{array}{l}\text { Brown-Jennings(1989), } \\
\text { Kim-Verrecchia(1991,1994) }\end{array}$ \\
\hline$\kappa \rightarrow \infty$ & $\theta_{w}+\varepsilon_{i}$ & $\theta_{w}+\eta$ & $\theta_{w}$ & $\approx \theta_{w}+u_{2}$ & He-Wang (1995) \\
\hline$\omega \rightarrow \infty$ & $\theta_{k}+\varepsilon_{i}$ & $\theta_{s}+\eta$ & $\theta_{k}+\theta_{s}$ & $\approx \theta_{s}+u_{2}$ & independent information sets \\
\hline
\end{tabular}

When $\kappa \rightarrow \infty$, the non-common factors, $\theta_{s}, \theta_{k}$ lose their importance and we end up in a one-factor structure with $\theta_{w}$ only. When also $\delta_{2} \rightarrow \infty$, second period price, $p_{2}$, will be fully revealing, so Londoners will behave as if they could liquidate for $\theta_{w}$, which is the only relevant factor remaining. Hence, in the case of $\kappa, \delta_{2} \rightarrow \infty$, effectively we have a two period model with one factor as in Brown and Jennings. When $\delta_{2}$ is finite, the model resembles to that of He and Wang (1995) as the liquidation value for Londoners, $p_{2}$, will contain the random term $u_{2}$ as well. However, when only $\omega \rightarrow \infty$, we have a different structure, very similar to Example 1, where private information sets of Londoners and New Yorkers get separated. As we will show in the next section: the larger $\omega$, the larger the effect of increasing disagreement.

\subsection{Analytical results}

In the first part of this section, we show that equilibrium of the model exists. In the second part, we present results on the equilibrium volume. 


\subsubsection{Equilibrium and existence}

We search for a linear equilibrium, so we assume that prices are given by the functions

$$
\begin{aligned}
& p_{2}=c_{2} y+b_{2}\left(\theta_{s}+\theta_{w}\right)+f_{2} q_{1}+g_{2} q_{0}-e_{2} u_{2} \\
& p_{1}=c_{1} y+b_{1}\left(\theta_{k}+\theta_{w}\right)+f_{1} q_{0}-e_{1} u_{1} \\
& p_{0}=b_{0}\left(\theta_{k}+\theta_{w}\right)-e_{0} u_{0}
\end{aligned}
$$

where $c_{t}, b_{t}, e_{t}, f_{1}, f_{2}, g_{2}$ are undetermined coefficients, while $q_{1}, q_{0}$ are specified below. Prices together with past prices and the public information are informationally equivalent with the following price signals

$$
\begin{aligned}
q_{2} & =\frac{1}{b_{2}}\left(p_{2}-c_{2} y-f_{2} q_{1}-g_{2} q_{0}\right)=\left(\theta_{s}+\theta_{w}\right)-\frac{e_{2}}{b_{2}} u_{2} \\
q_{1} & =\frac{1}{b_{1}}\left(p_{1}-c_{1} y-f_{1} q_{0}\right)=\left(\theta_{k}+\theta_{w}\right)-\frac{e_{1}}{b_{1}} u_{1} \\
q_{0} & =\frac{1}{b_{0}} p_{0}=\left(\theta_{k}+\theta_{w}\right)-\frac{e_{0}}{b_{0}} u_{0} .
\end{aligned}
$$

Below, we will show that the equilibrium trading activity is determined only by the noisiness of these price signals, so we define $\tau_{t}^{2}$ as the precision of $q_{t}$ :

$$
\frac{1}{\tau_{t}^{2}}=\frac{e_{t}^{2}}{b_{t}^{2} \delta_{t}^{2}} \text { or } \frac{\tau_{t}}{\delta_{t}}=\frac{b_{t}}{e_{t}} .
$$

Let us also define the following coefficients of variables in traders' information sets in different conditional expectations of New Yorkers and Londoners:

$$
\begin{aligned}
E\left(\theta \mid z_{j}, y, q_{2}, q_{1}, q_{0}\right) & =\bar{b} z_{j}+\bar{c} y+\bar{e} q_{2}+\bar{f} q_{1}+\bar{g} q_{0} \\
E\left(\theta_{s}+\theta_{w} \mid x_{i}, y, q_{1}, q_{0}\right) & =b_{s} x_{i}+c_{s} y+e_{s} q_{1}+f_{s} q_{0} \\
E\left(\theta \mid x_{i}, q_{0}\right) & =E\left(\theta_{k}+\theta_{w} \mid x_{i}, q_{0}\right)=E\left(q_{1} \mid x_{i}, q_{0}\right)=E\left(y \mid x_{i}, q_{0}\right)=b_{y} x_{i}+e_{y} q_{0} .
\end{aligned}
$$

From standard results (e.g. Brown and Jennings, 1989), we know that demand functions of New Yorkers in period 2 and Londoners in period 1 will be

$$
\begin{aligned}
d_{2}^{j} & =\frac{E\left(\theta \mid z_{j}, y, p_{2}, q_{1}, q_{0}\right)-p_{2}}{\operatorname{avar}\left(\theta \mid z_{j}, y, p_{2}, q_{1}, q_{0}\right)}=\frac{\bar{c} y+\bar{b} x_{j}+\bar{f} q_{1}+\bar{e} q_{2}+\bar{g} q_{0}-p_{2}}{\operatorname{avar}\left(\theta \mid z_{j}, y, p_{2}, q_{1}, q_{0}\right)} \\
d_{1}^{i} & =\frac{E\left(p_{2} \mid x_{i}, y, p_{1}, q_{0}\right)-p_{1}}{\operatorname{avar}\left(p_{2} \mid x_{i}, y, p_{1}, q_{0}\right)}=\frac{c_{2} y+b_{2}\left(b_{s} x_{i}+c_{s} y+e_{s} q_{1}+f_{s} q_{0}\right)+f_{2} q_{1}+g_{2} q_{0}-p_{1}}{\operatorname{avar}\left(p_{2} \mid x_{i}, y, p_{1}, q_{0}\right)}
\end{aligned}
$$

Finding the demand function in period 0 is a bit more subtle. Londoners maximize the following 
expected utility in period 0 :

$$
E\left(-\exp \left(-a\left(p_{1}-p_{0}\right) d_{1}-\frac{E\left(p_{2} \mid q_{1}, y, x_{j}, q_{0}\right)-p_{1}}{\operatorname{avar}\left(p_{2} \mid q_{1}, y, x_{j}, q_{0}\right)}\left(p_{2}-p_{1}\right)\right) \mid x_{i}, q_{0}\right)
$$

The source of the difficulty is that there are two random variables in this expression, $p_{1}$ (or $q_{1}$ ) and $y$. In the appendix, we show that as the outcome of this maximization - the demand function in period $0-$ is

$$
d_{0}^{i}=\frac{\left(E\left(p_{1} \mid x_{i}, q_{0}\right)-p_{0}\right)\left(\sigma_{q} s+1\right)+b_{s} E\left(d_{1}^{i} \mid x_{i}, q_{0}\right)\left(c_{1} \sigma_{y q}+b_{1} \sigma_{q}\right)}{a\left(c_{1}^{2} \sigma_{y}+c_{1}^{2} s \sigma_{y} \sigma_{q}-c_{1}^{2} s \sigma_{y q}^{2}+\sigma_{q} b_{1}^{2}+2 c_{1} \sigma_{y q} b_{1}\right)}
$$

where

$$
\left(\begin{array}{cc}
\sigma_{y} & \sigma_{y q} \\
\sigma_{y q} & \sigma_{q}
\end{array}\right)=\operatorname{var}\left(\left(\begin{array}{c}
y \\
q_{1}
\end{array}\right) \mid x_{i}, q_{0}\right)
$$

is the variance-covariance matrix of $y$ and $q_{1}$ conditional on a London-trader's information set in period 0. Intuitively, the first part in expression (12) represents the short-term demand component, while the second part represents the hedging component for demand in period 1.

We can show that in equilibrium, demand functions can be characterized completely by the equilibrium values of $\tau_{t}$ in the following manner:

$$
\begin{aligned}
d_{2}^{j} & =\frac{\tau_{2}}{\delta_{2}}\left(z_{j}-q_{2}\right)=\frac{\tau_{2}}{\delta_{2}} \varepsilon_{j}+u_{2} \\
d_{1}^{i} & =\frac{\tau_{1}}{\delta_{1}}\left(x_{i}-q_{1}\right)=\frac{\tau_{1}}{\delta_{1}} \varepsilon_{i}+u_{1} \\
d_{0}^{i} & =\frac{\tau_{0}}{\delta_{0}}\left(x_{i}-q_{0}\right)=\frac{\tau_{0}}{\delta_{0}} \varepsilon_{i}+u_{0} .
\end{aligned}
$$

The right hand sides of the three equations show that in each period, total positions consist of two parts. There is a risk-sharing part, $u_{t}$, which is purchased by each agent regardless of her information, and there is a speculative part, $\frac{\tau_{t}}{\delta t} \varepsilon_{i}$, which depends on the difference between the agent's signal and the true value of the factor, $\varepsilon_{i}$ or $\varepsilon_{j}$, and the coefficient $\frac{\tau_{t}}{\delta t}$. It is apparent that $\frac{\tau_{t}}{\delta t}$ determines how intensively the trader uses her private information to bet against the others, so we will label this fraction as trading intensity in period $t$. In particular, an optimistic trader will hold a positive position if $\frac{\tau_{t}}{\delta t}$ is positive and hold a negative position of $\frac{\tau_{t}}{\delta t}$ is negative. So trading activity and volume will depend how these coefficients change across periods. Here, we only present the steps which lead to (13). Expressions (14) and (15) are obtained very similarly.

From (10), the market clearing condition is

$$
D_{2}=\frac{\bar{c} y+\bar{b}\left(\theta_{s}+\theta_{w}\right)+\bar{f} q_{1}+\bar{e} q_{2}+\bar{g} q_{0}-p_{2}}{\operatorname{avar}\left(\theta \mid z_{j}, y, p_{2}, q_{1}, q_{0}\right)}=u_{2} .
$$

Using (6) and rearranging gives

$$
\bar{c} y+\bar{b}\left(\theta_{s}+\theta_{w}\right)+\bar{f} q_{1}+\bar{e} q_{2}+\bar{g} q_{0}-\operatorname{avar}\left(\theta \mid z_{j}, y, p_{2}, q_{1}, q_{0}\right) u_{2}=b_{2} q_{2}+c_{2} y+f_{2} q_{1}+g_{2} q_{0} .
$$

As the two sides have to be equal in equilibrium for any realizations of $u_{1}, u_{2}$ and $\eta$, 


$$
c_{2}=\bar{c}, \quad f_{2}=\bar{f}, \text { and } \quad g_{2}=\bar{g}
$$

must hold. This implies

$$
\bar{b} \frac{e_{2}}{b_{2}}\left(\theta_{s}+\theta_{w}\right)-\operatorname{avar}\left(\theta \mid z_{j}, y, p_{2}, q_{1}, q_{0}\right) \frac{e_{2}}{b_{2}} u_{2}=\left(b_{2}-\bar{e}\right) \frac{e_{2}}{b_{2}} q_{2} .
$$

As $q_{2}=\theta_{s}+\theta_{w}-\frac{e_{2}}{b_{2}} u_{2}$, this gives us

$$
\frac{\bar{b}}{\operatorname{avar}\left(\theta \mid z_{j}, y, p_{2}, q_{1}, q_{0}\right)}=\frac{b_{2}}{e_{2}}=\frac{\left(b_{2}-\bar{e}\right)}{\operatorname{avar}\left(\theta \mid z_{j}, y, p_{2}, q_{1}, q_{0}\right)},
$$

consequently,

$$
b_{2}=\bar{b}+\bar{e}
$$

Note, that expressions (16) and (17) determine the equilibrium value of the coefficients in the price function,(5), in terms of coefficients of the conditional expectation of $\theta$. If we substitute out $p_{2}, c_{2}, g_{2}, f_{2}$ from the left hand side of $(10)$, we get

$$
E\left(\theta \mid z_{j}, y, p_{2}, q_{1}, q_{0}\right)-p_{2}=b_{2}\left(z_{j}-q_{2}\right),
$$

which - together with the definition of $\tau_{2}$ and $q_{2}$ - implies

$$
d_{2}^{i}=\frac{\bar{b}\left(z_{j}-q_{2}\right)}{\operatorname{avar}\left(\theta \mid z_{j}, y, p_{2}, q_{1}, q_{0}\right)}=\frac{\tau_{2}}{\delta_{2}}\left(z_{j}-q_{2}\right)=\frac{\tau_{2}}{\delta_{2}} \varepsilon_{j}+u_{2} .
$$

Very similar steps applied to (11) and (12) gives us

$$
\begin{aligned}
& d_{1}^{i}=\frac{1}{a b_{2}} \frac{b_{s}}{\operatorname{var}_{i}\left(\theta_{s}+\theta_{k}\right)+\frac{1}{\tau_{2}^{2}}}\left(x_{i}-q_{1}\right)=\frac{\tau_{1}}{\delta_{1}}\left(x_{i}-q_{1}\right)=\frac{\tau_{1}}{\delta_{1}} \varepsilon_{i}+u_{1} \\
& d_{0}^{i}=\frac{\left(\sigma_{q} s+1\right)\left(\left(c_{1}+b_{1}\right) b_{y}+s\left(1-b_{y}\right) \frac{c_{1} \sigma_{y q}+b_{1} \sigma_{q}}{\sigma_{q} s+1}\right)}{a\left(c_{1}^{2} \sigma_{y}+c_{1}^{2} s \sigma_{y} \sigma_{q}-c_{1}^{2} s \sigma_{y q}^{2}+\sigma_{q} b_{1}^{2}+2 c_{1} \sigma_{y q} b_{1}\right)}\left(x_{i}-q_{0}\right)=\frac{\tau_{0}}{\delta_{0}}\left(x_{i}-q_{0}\right)=\frac{\tau_{0}}{\delta_{0}} \varepsilon_{i}+u_{0}
\end{aligned}
$$

From this procedure - similar to expressions (16) and (17) - we also gain expressions for $c_{1}$ and $b_{1}$ in terms of coefficients of the conditional expectations (7)- $(9)^{13}$. All of these, together with the expectational coefficients are given in the appendix. The last step is to find the equilibrium trading intensities, $\frac{\tau_{t}}{\delta_{t}}$, which give the equilibrium demand functions. For this, we simply plug in expressions for $b_{s}, \bar{b}, b_{2}$ and conditional variances into (18) and (19) and equate the coefficients of $\left(z_{j}-q_{2}\right),\left(x_{i}-q_{1}\right)$ and $\left(x_{i}-q_{0}\right)$ in the two sides of the equations (18)-(20). This gives a system of three equations with the three unknowns of $\tau_{1}, \tau_{2}, \tau_{0}$ :

$$
\tau_{2}=f^{2}\left(\tau_{2}, \tau_{1}, \tau_{0}\right)
$$

\footnotetext{
${ }^{13}$ Actually, we also obtain similar expressions for the other coefficients in the price functions $-e_{2}, f_{1}, e_{1}, b_{0}, e_{0}-$ but as they are not relevant for our purposes, we omit them to save space.
} 


$$
\begin{aligned}
\tau_{1} & =\left(\kappa^{2}-\omega \beta\right) f^{1}\left(\tau_{2}, \tau_{1}, \tau_{0}\right) \\
\tau_{0} & =f^{0}\left(\tau_{2}, \tau_{1}, \tau_{0}\right) .
\end{aligned}
$$

where $f^{0}\left(\tau_{2}, \tau_{1}, \tau_{0}\right), f^{1}\left(\tau_{2}, \tau_{1}, \tau_{0}\right)$ and $f^{2}\left(\tau_{2}, \tau_{1}, \tau_{0}\right)$ are well defined functions and both $f^{1}\left(\tau_{2}, \tau_{1}, \tau_{0}\right)$ and $f^{2}\left(\tau_{2}, \tau_{1}, \tau_{0}\right)$ are positive for any $\tau_{2}, \tau_{1}, \tau_{0}$. Their exact form is given in the appendix. It is easy to check that the corresponding equilibrium intensities when there is no announcement will be given by the same equations by setting $\beta=0$. When it could cause misunderstanding, we will distinguish between $\tau_{2}, \tau_{1}, \tau_{0}$ of the announcement and the no-announcement cases by the subscript $n$, for noannouncement. Furthermore, it is apparent for the announcement case that $\tau_{1}$ will be negative when $\omega$ is large: there will be belief swap. So in the latter case optimistic early traders will hold a positive position before the announcement $\left(\tau_{0}^{n}>0\right)$, but in the case of an announcement they will sell massively, ending up with a negative position $\left(\tau_{1}<0\right)$. We will return to the intuition of this case in the next section.

Hence, the equilibrium exists if and only if this system of equations has a fix point. The following proposition states that this will be the case for any parameter values.

Theorem 1 (Existence) From (21)-(22) any equilibrium is a fixed-point of a system:

$$
\tau_{2}=f^{2}\left(\tau_{2}, \tau_{1}, \tau_{0}\right), \quad \tau_{1}=\left(\kappa^{2}-\omega \beta\right) f^{1}\left(\tau_{2}, \tau_{1}, \tau_{0}\right), \quad \tau_{0}=f^{0}\left(\tau_{2}, \tau_{1}, \tau_{0}\right)
$$

There is always at least one equilibrium of this system both for the announcement and the noannouncement cases.

Proof. The proof is in the Appendix.

\subsubsection{Announcement and volume}

As the focus of this paper is the effect of announcement on trading volume, we will be interested in the change of volume in period 1 due to the announcement. From equations (11) and (12), the amount of trading of trader $i$ in period 1 will be given by

$$
v_{1}^{i}=d_{1}^{i}-d_{0}^{i}=\left(\frac{\tau_{1}}{\delta_{1}}-\frac{\tau_{0}}{\delta_{0}}\right) \varepsilon_{i}-u_{0}+u_{1} .
$$

Just as total positions, the total amount of trade of individual $i$ consist of two parts. There is an information-independent risk-sharing part, $u_{1}-u_{0}$, and there is a speculative part $\left(\frac{\tau_{1}}{\delta_{1}}-\frac{\tau_{0}}{\delta_{0}}\right) \varepsilon_{i}$, which is determined by the difference of trading intensities in the two periods and the private information of the trader. If we aggregate across traders, we get the following expression for total volume in period 


$$
\begin{aligned}
V_{1}=\frac{1}{2} \int\left|d_{1}^{i}-d_{0}^{i}\right| & d i=\frac{1}{2} \int_{\left(\frac{\tau_{1}}{\delta_{1}}-\frac{\tau_{0}}{\delta_{0}}\right) \varepsilon_{i}>u_{0}-u_{1}}\left(\left(\frac{\tau_{1}}{\delta_{1}}-\frac{\tau_{0}}{\delta_{0}}\right) \varepsilon_{i}+u_{1}-u_{0}\right) \phi\left(\alpha \varepsilon_{i}\right) d \varepsilon_{i}- \\
- & \frac{1}{2} \int_{\left(\frac{\tau_{1}}{\delta_{1}}-\frac{\tau_{0}}{\delta_{0}}\right) \varepsilon_{i}<u_{0}-u_{1}}\left(\left(\frac{\tau_{1}}{\delta_{1}}-\frac{\tau_{0}}{\delta_{0}}\right) \varepsilon_{i}+u_{1}-u_{0}\right) \phi\left(\alpha \varepsilon_{i}\right) d \varepsilon_{i}= \\
= & \left|\left(\frac{\tau_{1}}{\delta_{1}}-\frac{\tau_{0}}{\delta_{0}}\right)\right| \frac{1}{\sqrt{\alpha}} \phi(T)+\operatorname{sgn}\left(\frac{\tau_{1}}{\delta_{1}}-\frac{\tau_{0}}{\delta_{0}}\right)\left(u_{1}-u_{0}\right) \frac{1}{2}(1-2 \Phi(T))
\end{aligned}
$$

with $T=\alpha \frac{u_{0}-u_{1}}{\left(\frac{\tau_{1}}{\delta_{1}}-\frac{\tau_{0}}{\delta_{0}}\right)}$, where we used the result that if $\zeta \sim N\left(\mu, \sigma^{2}\right)$, then

$$
\int_{\zeta>L} \zeta \frac{\phi\left(\frac{\zeta-\mu}{\sigma}\right)}{\Phi(\alpha)} d \zeta=E(\zeta \mid \zeta>L)=\mu+\sigma \lambda(\alpha)
$$

with $\lambda(\alpha)=\frac{\phi(\alpha)}{1-\Phi(\alpha)}$ and $\alpha=\frac{L-\mu}{\sigma}$.

Hence, aggregate volume depends only on the realization of $u_{1}-u_{0}$, the precision of the private signals $\alpha$, and the distance between trading intensities, $\left|\left(\frac{\tau_{1}}{\delta_{1}}-\frac{\tau_{0}}{\delta_{0}}\right)\right|$. As the first one is unrelated to information or announcements, we will focus on the speculative volume, which we define as the volume when there is no risk-sharing trade:

$$
V_{1}^{S}=\left.V_{1}\right|_{u_{0}=u_{1}}=\left|\frac{\tau_{1}}{\delta_{1}}-\frac{\tau_{0}}{\delta_{0}}\right| \frac{1}{\sqrt{\alpha 2 \pi}}
$$

It must be clear now that - for results on the effect of announcement on speculative volume - we only have to compare the change in trading intensities in the announcement case, $\left|\frac{\tau_{1}}{\delta_{1}}-\frac{\tau_{0}}{\delta_{0}}\right|$, and the no-announcement case, $\left|\frac{\tau_{1}^{n}}{\delta_{1}}-\frac{\tau_{0}^{n}}{\delta_{0}}\right|$. The main result of this paper is that the outcome of this comparison will depend heavily and systematically on the information structure i.e. on the relative importance of the common factor, $\theta_{w}$, and the individual factors $\theta_{s}, \theta_{k}$. The following proposition shows that as individual factors become less important and the common factor becomes more important, volume disappears. This result is in line with our earlier observation that in a rational model with one factor trading volume around announcements is small, because the effects of increasing precision of opinions and decreasing disagreement cancel out.

Proposition 4 As $\delta_{2}, \kappa \rightarrow \infty$

$$
\frac{\tau_{2}}{\delta_{2}}=\frac{\tau_{1}}{\delta_{1}}=\frac{\tau_{0}}{\delta_{0}}=\frac{\tau_{2}^{n}}{\delta_{2}}=\frac{\tau_{1}^{n}}{\delta_{1}}=\frac{\tau_{0}^{n}}{\delta_{0}}=\frac{\alpha}{a},
$$

hence $V_{1}=V_{1}^{n}=0$ in this limit.

Proof. For $\frac{\tau_{2}}{\delta_{2}}, \frac{\tau_{1}}{\delta_{1}}, \frac{\tau_{2}^{n}}{\delta_{2}}$ and $\frac{\tau_{1}^{n}}{\delta_{1}}$, the result comes from the simple observation that the ordered limit

$$
\lim _{\delta_{2} \rightarrow \infty} \lim _{\kappa \rightarrow \infty} f^{2}\left(\tau_{2}, \tau_{1}, \tau_{0}\right)=\frac{\alpha}{a}
$$


and after substitution of $\tau_{2}=\frac{\alpha}{a}$

$$
\lim _{\delta_{2} \rightarrow \infty} \lim _{\kappa \rightarrow \infty} f^{1}\left(\tau_{2}, \tau_{1}, \tau_{0}\right)=\frac{\alpha}{a}
$$

The result for $\frac{\tau_{0}}{\delta_{0}}$ and $\frac{\tau_{0}^{n}}{\delta_{0}}$ can be obtained in a similar, but much more tedious way, if we take the limit of all the building-blocks of $f^{0}\left(\tau_{2}, \tau_{1}, \tau_{0}\right)$ and plug them in.

We confront this result with the next proposition, where we show that if one measures the effect of announcement by the proportion of volume in the announcement case to volume in the noannouncement case, this proportion will be arbitrary large as the common factor, $\theta_{w}$, loses its importance. The same is true for the proportion of speculative positions in both periods.

Proposition 5 If $\omega$ is large enough $D_{1}^{s}=\frac{1}{2} \int\left|\frac{\tau_{1}}{\delta_{0}} \varepsilon_{i}\right| d i>\frac{1}{2} \int\left|\frac{\tau_{1}^{n}}{\delta_{0}} \varepsilon_{i}\right| d i=D_{1}^{n}$ and $V_{1}>V_{1}^{n}$ and as $\omega \rightarrow \infty, \frac{D_{1}}{D_{1}^{n}} \rightarrow \infty, \frac{D_{0}}{D_{0}^{n}} \rightarrow \infty$. Furthermore, $\frac{V_{1}}{V_{1}^{n}} \rightarrow \infty$ (for almost all parameters).

Proof. The proof is in the appendix.

The intuition of this result is the same as in Example 1. Similar to the example, where two independent factors determined the quality of the restaurant, let us suppose here that $\omega$ is large, so the common factor, $\theta_{w}$ is unimportant, i.e. only the individual factors, $\theta_{s}$ and $\theta_{k}$ matter. Traders can bet only on those variables that are not part of the public information set. From the Londoners point of view in period 1 (the second period when they trade), the only variable in $p_{2}$ which is not part of the public information set - apart from the noise, $u_{2}-$ is $\theta_{s}$. But first period traders have no information on $\theta_{s}$, only on $\theta_{k}$. Hence, they will agree that they do not know anything (i.e. their guess will be the a priori mean, 0 ), and there will be agreement and no speculative trade. But if Londoners do not trade on their private information, their private information cannot be channelled into prices, so $p_{1}$ will be pure noise. So if we go back one period, in period 0 , Londoners should bet on $p_{1}$ and $p_{2}$, but they do not have any information neither on $p_{1}$, as it is pure noise, nor on $p_{2}$, because they do not know anything about $\theta_{s}$. Hence, there will be no speculative trade in period 0 either. It means that speculative volume, the difference between individual speculative positions in period 0 and period 1 , will also be zero. However, with public announcement the situation changes. Public announcement is $y \approx \theta_{s}+\theta_{k}+\eta$, if $\theta_{w}$ is unimportant. So Londoners will have some information on the sum of $\theta_{s}$ and $\theta_{k}$. But together with their private information on $\theta_{k}$, it gives them some information on $\theta_{s}$. What is more is that as they have different guesses on $\theta_{k}$ due to their different private signals, their guesses on $\theta_{s}$ will also be different. Therefore, public announcement increases disagreement. In particular, an early trader with a high private signal, will overestimate $\theta_{k}$, so for a given $y \approx \theta_{s}+\theta_{k}+\eta$, will under estimate $\theta_{s}$. This is why, the observation of the public signal will make her to hold a negative position i.e. to sell assets. With the opposite logic as in the no-announcement case, there will be trade in all periods and there will be volume.

\subsection{Numerical Results}

We calculated numerically the fix point of the system (21)-(22) with several parameter combinations. A typical graph of trading intensities $\frac{\tau_{t}}{\delta_{t}}$ and $\frac{\tau_{t}^{n}}{\delta_{t}}$ is shown in Figure 1. As we mentioned, the absolute 


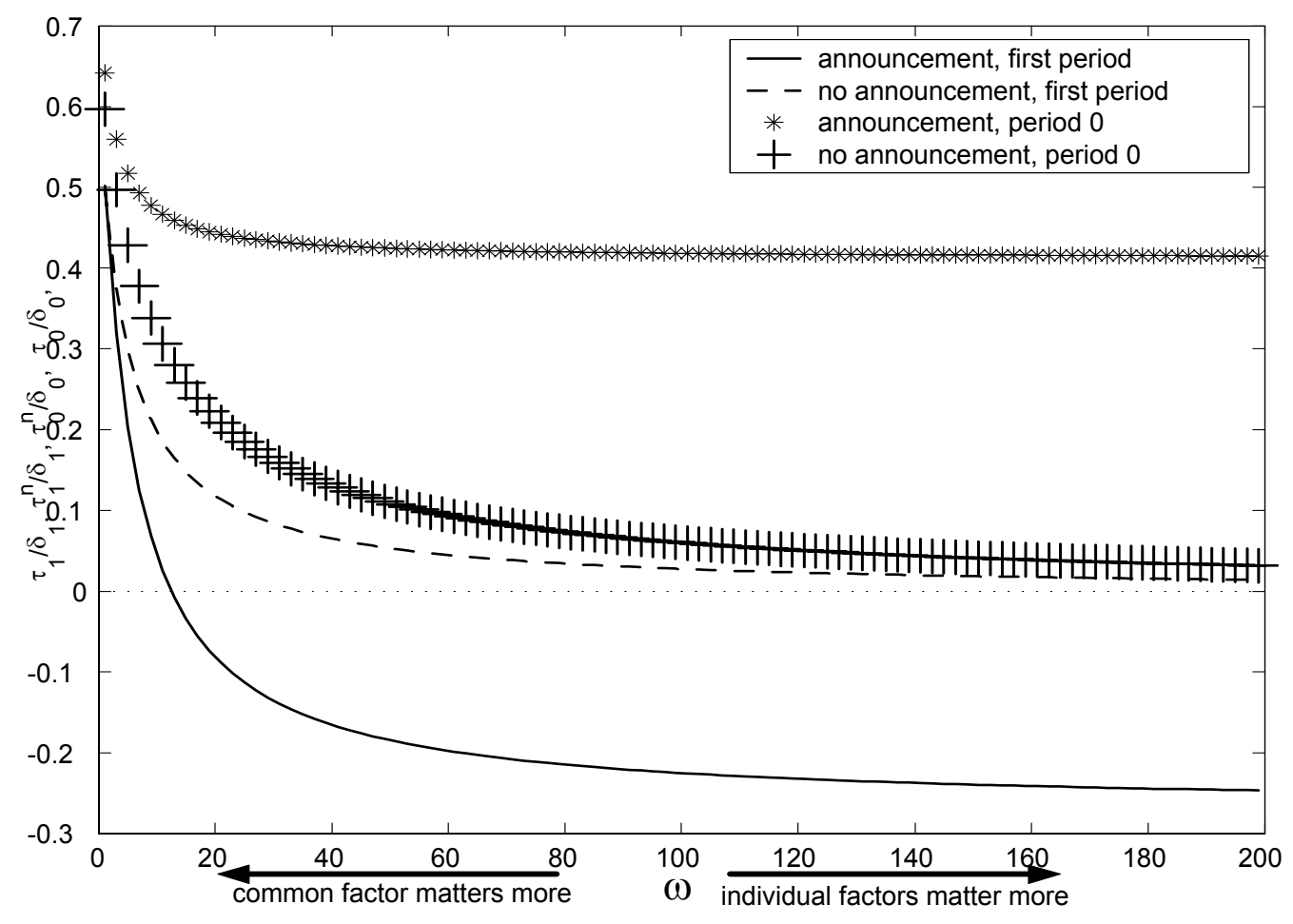

Figure 1: Trading intensities in periods 0 and 1 in the cases of announcement and no-announcement (the coefficient of $\varepsilon_{i}$ in the expression $d_{t}^{i}$ ) as the information set of traders across groups becomes more separated i.e. $\omega$ increases. Parameter values are $\delta_{2}=\kappa=5, \alpha=\beta=\delta_{1}=\delta_{0}=1$.

size of these coefficients is proportional to the absolute size of positions of our traders, while their sign shows the shows whether an optimistic trader desires to hold a positive or a negative position. The middle two lines are $\frac{\tau_{0}^{n}}{\delta_{0}}$ and $\frac{\tau_{1}^{n}}{\delta_{1}}$ (the trading intensity in the no-announcement case in period 1 and 0 ). Both goes to 0 as $\omega \rightarrow \infty$ as it is stated in the proof of Proposition 5 and in line with our intuitive story when $\theta_{w}$ is unimportant. With announcement, in period 0 traders bet intensively on the size of $y$ based on their private information (line with asterisk), and in period 1 they bet intensively on $p_{2}$ (solid line). The larger the distance between the two lines, the larger the trading volume around announcements. When $\omega$ is small, we are close to the standard information case (one factor model). It is apparent that at this extreme, all lines are almost equal, so trading volume is small. If $\kappa$ and $\delta_{2}$ would be large enough, all lines would coincide as it is stated in Proposition 4 . It is spectacular that as $\omega$ grows - private information sets become separated across groups - the lines corresponding to the announcement cases fan out. This shows the potential of our story in explaining the jump in trading volume around announcements. The change of the sign of the solid line shows, how the desired position of an optimistic early trader changes after the announcement. When the common component is important, her optimistic view on the common component dominates her pessimistic view about $\theta_{s}$. As the importance of the common component disappears, the second effect will dominate, just as in Example 1 or just as we described in the previous section. In this case trading volume will be particularly high, because early traders with high signals change their long position to short position 
(from the stared line to the solid line) as a result of the announcement across periods 0 and 1 , while early traders with low private signals do the opposite.

\section{Confrontation to stylized facts from the empirical literature}

In this section, we will confront our results to empirical evidence on the effect of public announcements on trading activity. Firstly, we consider well-established results from high-frequency data and the puzzle of increasing trading without price changes. Then we turn briefly to the work of Bamber et al (1997), which is of special interest for us as it attempts to connect the change in trading activity directly to measures of disagreement.

Recent evidence from high-frequency data-sets (Evans and Lyons, 2001,2003, Love and Payne, 2003, Love 2004, Fleming and Remolona, 1999) show a prolonged intense trading period after announcements when

1. there is a simultaneous increase in buying orders and selling orders ${ }^{14}$

2. order flows, the difference between buying and selling orders, are more volatile

3. order flows are more informative i.e. they influence price formation more.

Let us include also a fourth stylized fact which Kandel and Person (1995) found inconsistent with rational models on the basis of the model of Kim and Verrecchia $(1991,1994)$.

4. There is a jump in trading volume due to the announcement, even if there is no corresponding price change.

Our rational expectations model is of a reduced form. All traders submit whole demand schedules and orders are executed on the market-clearing price. Hence, in our model there is no order flow. In reality, a market maker neither observes whole demand schedules nor observes all of them at the same time. She has to map them through time. She quotes a price that is good for any amount, some traders make transactions on this price, and the market-maker updates her quote depending on the received orders. Order flow is like the aggregate trades of a small group of traders executed at a closeto-equilibrium price. We believe that the nearest we can get in our model to the behaviour of order flow, is to consider the behaviour of individual orders at equilibrium price, because the aggregation of a small number of trades must have similar characteristics to the parts of this aggregation. Firstly, we will argue that if one is willing to accept individual trades as a proxy for order flow, then our model is consistent with the three observed facts above.

\footnotetext{
${ }^{14}$ In the FX market, all dealers act as market makers, so all submit bid and ask prices simultaneously to the brokerage system, which are good for any amount. Then any of the dealers can initiate transactions on the best submitted quotes. A transaction is called a buying (selling) order, if the initiator of the transaction buys (sells) the commodity currency. Hence, the number of buys and the number of sells in any FX dataset can move independently of each other. (See Lyons, 2001, for detailed discussion on the microstructure of FX markets.)
} 
1. It is easy to see that both buys and sells increase due to announcement in our model as individual orders are given by

$$
d_{1}^{i}-d_{0}^{i}=\left(\frac{\tau_{1}}{\delta_{1}}-\frac{\tau_{0}}{\delta_{0}}\right) \varepsilon_{i}+u_{1}-u_{0}=\left(\frac{\tau_{1}}{\delta_{1}}-\frac{\tau_{0}}{\delta_{0}}\right) x_{i}-\left(\frac{\tau_{1}}{\delta_{1}} q_{1}-\frac{\tau_{0}}{\delta_{0}} q_{0}\right)
$$

and we showed that $\left(\frac{\tau_{1}}{\delta_{1}}-\frac{\tau_{0}}{\delta_{0}}\right)$ can be much larger when there is announcement, than when there is none.

2. Similarly, the volatility of individual orders depend positively on $\left|\frac{\tau_{1}}{\delta_{1}}-\frac{\tau_{0}}{\delta_{0}}\right|$ as well, since

$$
\operatorname{var}\left(d_{1}^{i}-d_{0}^{i}\right)=\left(\frac{\tau_{1}}{\delta_{1}}-\frac{\tau_{0}}{\delta_{0}}\right)^{2} \frac{1}{\alpha}+\frac{1}{\delta_{1}^{2}}+\frac{1}{\delta_{2}^{2}}
$$

3. Note, that in the mapping process of demand curves described above, the information content of individual orders depends on how strongly they are correlated with private information. It is so because the more heavily a trader uses her information on her speculative betting, the easier the market maker can deduct the private information of the trader. Since for a given price, $q_{1}$,

$$
\operatorname{cov}\left(d_{1}^{i}-d_{0}^{i}, x_{i}\right)=\left(\frac{\tau_{1}}{\delta_{1}}-\frac{\tau_{0}}{\delta_{0}}\right)
$$

The informativeness of prices again depends only on $\left(\frac{\tau_{1}}{\delta_{1}}-\frac{\tau_{0}}{\delta_{0}}\right)$.

For the fourth fact, let us just have another look to formula (23) for aggregate speculative volume:

$$
V_{1}^{S}=\left.V_{1}\right|_{u_{0}=u_{1}}=\left|\frac{\tau_{1}}{\delta_{1}}-\frac{\tau_{0}}{\delta_{0}}\right| \frac{1}{\sqrt{\alpha 2 \pi}}
$$

It should be clear that it is unrelated to price changes as speculative volume shows the flow of previously existing, but less relevant information into the market. Note also that, consistent with the effect of announcement when there is also a price change, price changes will be connected to aggregate volume through the risk sharing part of total volume.

Our formula for speculative volume also shows how our model is related to results in Bamber et al (1997). They show - using the proxy of changes in analysts' forecasts - that trading volume after announcements is positively related to three different aspects of disagreement: dispersion in prior beliefs, increase of dispersion after announcements, and the change of positions of investor's beliefs relative to each other: belief swap. It is apparent from (23) that speculative volume in our model is related to all these three aspects of disagreement. The positive dependence on $\frac{1}{\alpha}$ shows the effect of a priori dispersion of opinion, the term $\left|\frac{\tau_{1}}{\delta_{1}}-\frac{\tau_{0}}{\delta_{0}}\right|$ shows the effect of increasing disagreement and the fact that for large $\omega, \frac{\tau_{1}}{\delta_{1}}$ turns into negative shows that we will have belief swaps typically in those cases where there is higher trading volume. 


\section{Conclusion}

Increased trading volume after public announcements in financial markets are widely perceived as the sign of increasing disagreement due to the announcement. Hence, the same piece of public information has very different effects on the trading activity of different traders. In this paper, we showed - in contrast to the existing literature - that this stylized fact was an implication of a standard asymmetric information asset pricing model with common-priors if some traders were trading for resale. These early traders - instead of the fundamental value of the asset - were interested in the expectation of the fundamental value of future traders. Hence, even with a simple and plausible information structure, where higher values of private or public signals imply higher fundamental value, it is possible that a given public signal implies a higher average opinion of the future trader for one early trader but lower average opinion of the future trader for an other early trader. Therefore, disagreement increased across traders. We showed that this was the case when private information sets of traders arriving in different periods were only weakly connected. The intuition was that before the public announcement, early traders did not have too much information on future traders information, so their expectation on future traders expectation was very close to the a priori mean. However, public announcement - which was related to all information sets - connected early trader information to latecomers information, so informational differences across early traders became relevant. Consequently, the hectic trading activity after the announcement was a sign of the existing private information flowing into the market.

We see two promising routes for future research. In the last section, we argued that our findings are consistent with many stylized facts from the empirical literature. However, it would be intriguing to confront our model to further tests. Firstly, one could conduct other indirect tests to check whether the implication of our model is in line with the data. For example, our model implies that the effect of non-expected announcements to trading volume should be comparable to the effect of expected announcements. Although informational models are hard to test directly in general, it might be possible to search for latent informational factors behind trading and relating them to our UK and US factors.

The second route would be to generalize the theoretical implications of our model to other contexts. It is quite common in every field of informational economics that - mainly because of technical reasons - models use affiliated informational structures i.e. where higher values in one subset of random variables makes higher values in another subset more probable. In this paper, we partially relaxed this assumption. Although higher private and public signals implied higher fundamental value, higher private signals of some agents implied lower private signals for other agents. We showed that this departure had very interesting consequences in the financial context. Future research should explore consequences in other contexts like auction theory or industrial organization. 


\section{References}

[1] Adam, Klaus (2003): Optimal monetary policy with imperfect common knowledge, mimeo.

[2] Allen, Franklin - Stephen Morris - Hyun Song Shin (2003): Beauty Contests, Bubbles and Iterated Expectations in Asset Markets, mimeo.

[3] Amato, Jeffery D.- Hyun Song Shin (2003): Public and Private Information in Monetary Policy Models, mimeo.

[4] Bamber, Linda Smith - Orie E. Barron - Thomas L. Stober (1997): Trading Volume and Different Aspects of Disagreement Coincident with Earnings Announcements, Accounting Review, 72(4), 575-597.

[5] Brown, David P. - Robert H. Jennings (1989): On Technical Analysis, Review of Financial Studies, $2(4), 527-551$.

[6] Brunnermeier, Marcus (2001): Asset-pricing under Asymmetric Information - Bubbles, Crashes, Technical Analysis and Herding, Oxford University Press.

[7] Evans, Martin D. D. - Richard K. Lyons (2001): Why Order Flow Explains Exchange Rates, mimeo.

[8] Evans, Martin D.D. - Richard K. Lyons (2003): How Is Macro News Transmitted to Exchange Rates?, mimeo.

[9] Fleming, M. - E. Remolona (1999): Price formation and liquidity in the US treasury market, Journal of Finance, 54, 1901-1915.

[10] Foster, Douglas F. - S. Viswanathan (1996): Strategic trading when agents forecast the forecasts of others, Journal of Finance, 51(4), 1437-1478.

[11] Grossman, S. - J. Stiglitz (1980): On the impossibility of informationally efficient markets, American Economic Review, 70, 393-408.

[12] Harris, M. - A. Raviv (1993): Differences of opinion make a horse race, Review of Financial Studies, 6, 473-506.

[13] He, Hua - Jiang Wang (1995): Differential Information and Dynamic Behaviour of Stock Trading Volume, Review of Financial Studies, 8(4), pp. 919-972.

[14] Hellwig, Christian (2002): Public announcements, adjustment delays and the business cycle, mimeo.

[15] Jewitt, Ian (2004): Information acquisition and disclosure in auctions, mimeo.

[16] Kandel, Eugene - Neil D. Pearson (1995): Differential Interpretation of Public Signals and Trade in Speculative Markets, Journal of Political Economy, 103(4), 831-872. 
[17] Kim, Oliver - Robert E. Verrecchia (1991): Trading Volume and Price Reactions to Public Announcements, Journal of Accounting Research, 29(2), 302-321.

[18] Kim O. and R.E. Verrecchia 1994. Liquidity and volume around earning announcements, Journal of Accounting and Economics, 17(1-2), January, 41-67.

[19] Kim, Oliver - Robert E. Verrecchia (1997). Pre-announcement and event-period private information. Journal of Accounting and Economics 24 (3), 395-420

[20] Kondor, Péter (2004): Rational Trader Risk, mimeo.

[21] Love, Ryan (2004): First and second moment effects of macroeconomics news in high frequency foreign exchange data, mimeo.

[22] Love, Ryan - Richard Payne (2003): Macroeconomic news, order flows, and exchange rates, Discussion Paper 475, Financial Markets Group, London School of Economics.

[23] Lyons, Richard K. (2001): The Microstructure Approach to Exchange Rates, MIT Press, Boston.

[24] Milgrom, Paul R. (1981): Good News and Bad News: Representation Theorems and Applications, Bell Journal of Economics, 12(2), 380-391.

[25] Varian, Hal R. (1989): Differences of opinion in financial markets in C. Stone (eds): Financial risk: theory, evidence and implications, proceedings of the eleventh annual economic policy conference of the Federal Reserve Bank of St. Louis, Kluwer, Boston pp. 3-37.

[26] Woodford, Michael (2003): Imperfect Common Knowledge and the Effects of Monetary Policy, in P. Aghion, R. Frydman, J. Stiglitz and M. Woodford (eds) Knowledge, Information and Expectations in Modern Macroeconomics: In Honor of Edmund S. Phelps, Princeton University Press, Princeton, pp 25-58. 


\section{Appendix}

\section{A.1 Demand in period 0}

In period zero traders maximize the expected utility

$$
\begin{gathered}
E\left(-\exp \left(-a\left(p_{1}-p_{0}\right) d_{0}^{i}-\frac{E\left(p_{2} \mid q_{1}, y, x_{i}, q_{0}\right)-p_{1}}{\operatorname{avar}\left(p_{2} \mid q_{1}, y, x_{i}, q_{0}\right)}\left(p_{2}-p_{1}\right)\right) \mid x_{i}, q_{0}\right)= \\
=E\left(E\left(-\exp \left(-a\left(p_{1}-p_{0}\right) d_{0}^{i}-\frac{E\left(p_{2} \mid q_{1}, y, x_{i}, q_{0}\right)-p_{1}}{\operatorname{avar}\left(p_{2} \mid q_{1}, y, x_{i}, q_{0}\right)}\left(p_{2}-p_{1}\right)\right) q_{0}, y, x_{i}, q_{1}\right) \mid x_{i}, q_{0}\right)= \\
=E\left(-\exp \left(-a\left(p_{1}-p_{0}\right) d_{0}^{i}-\frac{\left(E\left(p_{2} \mid q_{1}, y, x_{i}, q_{0}\right)-p_{1}\right)^{2}}{2 \operatorname{var}\left(p_{2} \mid q_{1}, y, x_{j}, q_{0}\right)}\right) \mid x_{i}, q_{0}\right)= \\
=E\left(-\exp \left(-a\left(b_{1} q_{1}+c_{1} y+f_{1} q_{0}-p_{0}\right) d_{0}^{i}-\frac{1}{2} s\left(x_{i}-q_{1}\right)^{2}\right) \mid x_{i}, q_{0}\right)
\end{gathered}
$$

where

$$
s=\frac{b_{s}^{2}}{\left(\operatorname{var}\left(\theta_{s}+\theta_{w} \mid q_{1}, y, x_{j}, q_{0}\right)+\frac{1}{\tau_{2}^{2}}\right)}=b_{2} a \frac{\tau_{1}}{\delta_{1}} b_{s} .
$$

If we write the expression in the inner bracket into matrix form and we use the standard result for the expectation of exponentials with quadratic forms ${ }^{15}$, we get

$$
E\left(-\exp \left(\begin{array}{c}
-\frac{1}{2} s x_{i}^{2}-a\left(f_{1} q_{0}-p_{0}\right) d_{0}^{i}+\left(\begin{array}{cc}
-a c_{1} d_{0}^{i} & \left(-a b_{1} d_{0}^{i}+s x_{i}\right)
\end{array}\right)\left(\begin{array}{c}
y \\
q_{1}
\end{array}\right) \\
-\left(\begin{array}{cc}
y & q_{1}
\end{array}\right)\left(\frac{1}{2} s\right)\left(\begin{array}{cc}
0 & 0 \\
0 & 1
\end{array}\right)\left(\begin{array}{c}
y \\
q_{1}
\end{array}\right)
\end{array}\right) \mid x_{i}, q_{0}\right)=
$$

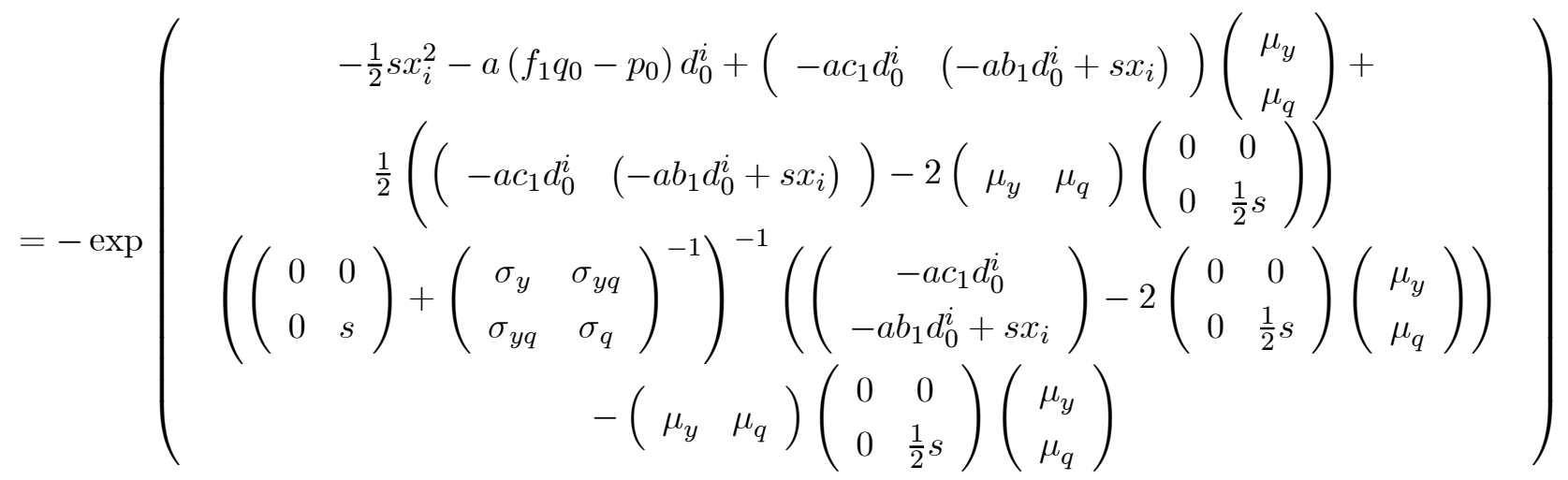

${ }^{15}$ If $c$ is constant scalar, $L$ is a $n x 1$ constant vector, $N$ is an $n x n$ constant matrix and $M$ is an $n x 1$ stochastic matrix and $I$ is an information set, then

$$
\begin{aligned}
& E\left(-\exp \left(c+L^{\prime} M-M^{\prime} N M^{\prime}\right) \mid I\right)= \\
& \left.\quad-|W|^{-1 / 2}\left|2 N+W^{-1}\right|^{-1 / 2} \exp \left(c+L^{\prime} Q-Q^{\prime} N Q+\frac{1}{2}\left(L^{\prime}-2 Q^{\prime} N\right)\right)\left(2 N+W^{-1}\right)^{-1}(L-2 N Q)\right)
\end{aligned}
$$

where $Q=E(M \mid I)$ and $W=\operatorname{var}(M \mid I)$ (see Brunnermeier, 2001, page 110). 
where

$$
\begin{aligned}
& \mu_{q}=E\left(q_{1} \mid x_{i}, q_{0}\right) \\
& \mu_{y}=E\left(y \mid x_{i}, q_{0}\right) .
\end{aligned}
$$

and

$$
\left(\begin{array}{cc}
\sigma_{y} & \sigma_{y q} \\
\sigma_{y q} & \sigma_{q}
\end{array}\right)=\operatorname{var}\left(\left(\begin{array}{c}
y \\
q_{1}
\end{array}\right) \mid x_{i}, q_{0}\right) .
$$

Maximizing the term in the bracket with respect to $d_{0}^{i}$ gives the demand function

$$
d_{0}^{i}=\frac{\left(c_{1} \mu_{y}+\mu_{q} b_{1}+q_{0} f_{1}-p_{0}\right)\left(\sigma_{q} s+1\right)}{a\left(c_{1}^{2} \sigma_{y}+c_{1}^{2} s \sigma_{y} \sigma_{q}-c_{1}^{2} s \sigma_{y q}^{2}+\sigma_{q} b_{1}^{2}+2 c_{1} \sigma_{y q} b_{1}\right)}+\frac{s\left(x_{i}-\mu_{q}\right)\left(c_{1} \sigma_{y q}+b_{1} \sigma_{q}\right)}{a\left(c_{1}^{2} \sigma_{y}+c_{1}^{2} s \sigma_{y} \sigma_{q}-c_{1}^{2} s \sigma_{y q}^{2}+\sigma_{q} b_{1}^{2}+2 c_{1} \sigma_{y q} b_{1}\right)}
$$

, which is identical with the one in the text. The second order condition of the maximization is

$$
\left(c_{1}^{2} \sigma_{y}+c_{1}^{2} s \sigma_{y} \sigma_{q}-c_{1}^{2} s \sigma_{y q}^{2}+\sigma_{q} b_{1}^{2}+2 c_{1} \sigma_{y q} b_{1}\right)>0 .
$$

\section{A.2 Expectations, variances and coefficients in the price-functions}

We give here the conditional expectations and variances obtained by standard results on normal variables (see e.g. Brunnermeier, 2001, p12). We also give the equilibrium expressions for coefficients in the price function. The method to obtain the latter is described in the text.

$$
\begin{aligned}
\sigma_{y} & =\frac{2 \kappa \omega \beta+\kappa^{2} \beta+\beta \alpha \omega+\beta \alpha \kappa+\beta \tau_{0}^{2} \omega+\beta \tau_{0}^{2} \kappa+\kappa \alpha \omega+\kappa^{2} \alpha+\kappa \tau_{0}^{2} \omega+\kappa^{2} \tau_{0}^{2}+\kappa^{2} \omega}{\left(\alpha \omega+\kappa \alpha+\tau_{0}^{2} \omega+\kappa \tau_{0}^{2}+\kappa \omega\right) \kappa \beta} \\
\sigma_{q} & =\frac{\tau_{1}^{2} \omega+\tau_{1}^{2} \kappa+\alpha \omega+\kappa \alpha+\tau_{0}^{2} \omega+\kappa \tau_{0}^{2}+\kappa \omega}{\left(\alpha \omega+\kappa \alpha+\tau_{0}^{2} \omega+\kappa \tau_{0}^{2}+\kappa \omega\right) \tau_{1}^{2}} \\
\sigma_{y q} & =\frac{\omega+\kappa}{\alpha \omega+\kappa \alpha+\tau_{0}^{2} \omega+\kappa \tau_{0}^{2}+\kappa \omega} \\
b_{y} & =(\omega+\kappa) \frac{\alpha}{\alpha \omega+\alpha \kappa+\tau_{0}^{2} \omega+\tau_{0}^{2} \kappa+\kappa \omega} \\
c_{1} & =\left(b_{2} c_{s}+\bar{c}\right) \\
b_{1} & =\left(b_{2}\left(b_{s}+e_{s}\right)+\bar{f}\right)
\end{aligned}
$$

where

$b_{2}=\frac{\left(\alpha \omega \tau_{0}^{2}+\alpha \omega \tau_{1}^{2}+\kappa \omega \alpha+\kappa \alpha \tau_{0}^{2}+\kappa \alpha \tau_{1}^{2}+\alpha \kappa^{2}+\tau_{0}^{2} \omega \tau_{2}^{2}+\tau_{1}^{2} \omega \tau_{2}^{2}+\kappa \omega \tau_{2}^{2}+\kappa \tau_{0}^{2} \tau_{2}^{2}+\kappa \tau_{1}^{2} \tau_{2}^{2}+\tau_{2}^{2} \kappa^{2}\right)}{B}$

with

$$
B=\left(\begin{array}{c}
\alpha \omega \beta+\kappa^{2} \beta+\tau_{1}^{2} \kappa^{2}+\tau_{2}^{2} \kappa^{2}+\alpha \kappa^{2}+\kappa^{2} \omega+\tau_{0}^{2} \kappa^{2}+\alpha \kappa \beta+\kappa \omega \tau_{1}^{2}+\tau_{1}^{2} \omega \tau_{2}^{2}+ \\
\kappa \omega \tau_{2}^{2}+2 \kappa \tau_{0}^{2} \tau_{2}^{2}+\tau_{0}^{2} \omega \tau_{2}^{2}+2 \kappa \tau_{1}^{2} \tau_{2}^{2}+\kappa \omega \tau_{0}^{2}+\kappa \omega \alpha+ \\
+\tau_{0}^{2} \kappa \beta+\tau_{1}^{2} \omega \beta+\tau_{1}^{2} \kappa \beta+\tau_{0}^{2} \omega \beta+\tau_{2}^{2} \beta \kappa+\tau_{1}^{2} \tau_{2}^{2} \beta+\tau_{0}^{2} \tau_{2}^{2} \beta+\tau_{2}^{2} \omega \beta+2 \kappa \omega \beta \\
+2 \kappa \alpha \tau_{0}^{2}+2 \kappa \alpha \tau_{1}^{2}+\alpha \omega \tau_{1}^{2}+\alpha \tau_{1}^{2} \beta+\alpha \omega \tau_{0}^{2}+\alpha \tau_{0}^{2} \beta
\end{array}\right)
$$


and

$$
\begin{aligned}
& \bar{f}=\frac{1}{B} \tau_{1}^{2}\left(\alpha+\tau_{2}^{2}+\kappa\right)(\omega+\kappa) \\
& \bar{c}=\frac{\beta\left(\tau_{0}^{2} \kappa+\tau_{0}^{2} \tau_{2}^{2}+\tau_{0}^{2} \omega+\alpha \tau_{0}^{2}+\alpha \tau_{1}^{2}+\alpha \omega+\kappa \alpha+\kappa^{2}+2 \kappa \omega+\kappa \tau_{2}^{2}+\omega \tau_{2}^{2}+\tau_{1}^{2} \tau_{2}^{2}+\tau_{1}^{2} \omega+\tau_{1}^{2} \kappa\right)}{B} \\
& c_{s}=\frac{\beta\left(\kappa+\tau_{1}^{2}+\alpha+\tau_{0}^{2}\right)(\omega+\kappa)}{\kappa^{2} \omega+2 \kappa \omega \beta+\kappa \omega \tau_{0}^{2}+\tau_{0}^{2} \kappa^{2}+\kappa^{2} \beta+\alpha \kappa^{2}+\kappa \omega \alpha+\kappa \omega \tau_{1}^{2}+\tau_{1}^{2} \kappa^{2}+\tau_{0}^{2} \kappa \beta+\tau_{1}^{2} \omega \beta+\tau_{1}^{2} \kappa \beta+\tau_{0}^{2} \omega \beta+\alpha \kappa \beta+\alpha \omega \beta} \\
& e_{s}=\frac{\tau_{1}^{2} \kappa^{2}-\tau_{1}^{2} \omega \beta}{\kappa^{2} \omega+2 \kappa \omega \beta+\kappa \omega \tau_{0}^{2}+\tau_{0}^{2} \kappa^{2}+\kappa^{2} \beta+\alpha \kappa^{2}+\kappa \omega \alpha+\kappa \omega \tau_{1}^{2}+\tau_{1}^{2} \kappa^{2}+\tau_{0}^{2} \kappa \beta+\tau_{1}^{2} \omega \beta+\tau_{1}^{2} \kappa \beta+\tau_{0}^{2} \omega \beta+\alpha \kappa \beta+\alpha \omega \beta} \\
& b_{s}=\frac{\alpha \kappa^{2}-\alpha \omega \beta}{\kappa^{2} \omega+2 \kappa \omega \beta+\kappa \omega \tau_{0}^{2}+\tau_{0}^{2} \kappa^{2}+\kappa^{2} \beta+\alpha \kappa^{2}+\kappa \omega \alpha+\kappa \omega \tau_{1}^{2}+\tau_{1}^{2} \kappa^{2}+\tau_{0}^{2} \kappa \beta+\tau_{1}^{2} \omega \beta+\tau_{1}^{2} \kappa \beta+\tau_{0}^{2} \omega \beta+\alpha \kappa \beta+\alpha \omega \beta} \\
& \operatorname{var}\left(\theta_{s}+\theta_{w} \mid x_{i}, q_{1}, q_{0}, y\right)=\quad \\
& =\frac{\tau_{1}^{2} \omega+2 \tau_{1}^{2} \kappa+\omega \beta+\kappa^{2}+\kappa \omega+\alpha \omega+2 \alpha \kappa+\tau_{1}^{2} \beta+\alpha \beta+2 \tau_{0}^{2} \kappa+\tau_{0}^{2} \omega+\beta \kappa+\tau_{0}^{2} \beta}{\kappa^{2} \omega+2 \kappa \omega \beta+\kappa \omega \tau_{0}^{2}+\tau_{0}^{2} \kappa^{2}+\kappa^{2} \beta+\alpha \kappa^{2}+\kappa \omega \alpha+\kappa \omega \tau_{1}^{2}+\tau_{1}^{2} \kappa^{2}+\tau_{0}^{2} \kappa \beta+\tau_{1}^{2} \omega \beta+\tau_{1}^{2} \kappa \beta+\tau_{0}^{2} \omega \beta+\alpha \kappa \beta+\alpha \omega \beta} \\
& \operatorname{var}\left(\theta \mid z_{j}, q_{2}, q_{1}, q_{0}, y\right)=\frac{\left(\tau_{0}^{2} \kappa+\tau_{0}^{2} \tau_{2}^{2}+\tau_{0}^{2} \omega+\alpha \tau_{0}^{2}+\alpha \tau_{1}^{2}+\alpha \omega+\kappa \alpha+\kappa^{2}+2 \kappa \omega+\kappa \tau_{2}^{2}+\omega \tau_{2}^{2}+\tau_{1}^{2} \tau_{2}^{2}+\tau_{1}^{2} \omega+\tau_{1}^{2} \kappa\right)}{B}
\end{aligned}
$$

\section{A.3 Proof of existence}

The equilibrium is given by the fixed point of the system

$$
\begin{aligned}
\tau_{2} & =f^{2}\left(\tau_{2}, \tau_{1}, \tau_{0}\right)= \\
& =\delta_{2} \frac{1}{a} \alpha \frac{\tau_{0}^{2} \omega+\tau_{1}^{2} \omega+\kappa \omega+\tau_{0}^{2} \kappa+\tau_{1}^{2} \kappa+\kappa^{2}}{\tau_{0}^{2} \kappa+\tau_{0}^{2} \tau_{2}^{2}+\tau_{0}^{2} \omega+\alpha \tau_{0}^{2}+\alpha \tau_{1}^{2}+\alpha \omega+\kappa \alpha+\kappa^{2}+2 \kappa \omega+\kappa \tau_{2}^{2}+\omega \tau_{2}^{2}+\tau_{1}^{2} \tau_{2}^{2}+\tau_{1}^{2} \omega+\tau_{1}^{2} \kappa} \\
\tau_{1} & =f^{1}\left(\tau_{2}, \tau_{1}, \tau_{0}\right)= \\
& =\delta_{1} \tau_{2}^{2} \alpha \frac{\kappa^{2}-\omega \beta}{a\left(\kappa \tau_{0}^{2} \tau_{2}^{2}+\kappa \omega \tau_{2}^{2}+\tau_{2}^{2} \kappa^{2}+\omega \tau_{1}^{2} \tau_{2}^{2}+2 \alpha \omega \tau_{2}^{2}+\kappa \tau_{1}^{2} \tau_{2}^{2}+2 \kappa \alpha \tau_{2}^{2}+\tau_{0}^{2} \omega \tau_{2}^{2}+\alpha \kappa^{2}+\kappa \omega \alpha\right)} \\
\tau_{0} & =f^{0}\left(\tau_{2}, \tau_{1}, \tau_{0}\right)=\frac{\delta_{0}\left(\sigma_{q} s+1\right)\left(c_{1}+b_{1}\right) b_{y}+s\left(1-b_{y}\right)\left(c_{1} \sigma_{y q}+b_{1} \sigma_{q}\right)}{a\left(c_{1}^{2} \sigma_{y}+c_{1}^{2} s \sigma_{y} \sigma_{q}-c_{1}^{2} s \sigma_{y q}^{2}+\sigma_{q} b_{1}^{2}+2 c_{1} \sigma_{y q} b_{1}\right)}
\end{aligned}
$$

We show the existence in three steps.

Lemma 1 Let us fix $\tau_{0}=\bar{\tau}_{0}$ at any arbitrary level. The system $\tau_{2}=f^{2}\left(\tau_{2}, \tau_{1}, \bar{\tau}_{0}\right), \tau_{1}=f^{1}\left(\tau_{2}, \tau_{1}, \bar{\tau}_{0}\right)$ will have at least one fix point, $\left(\tau_{1}^{*}, \tau_{2}^{*}\right)$. Additionally, $\tau_{2}^{\min } \leq \tau_{2}^{*}<\delta_{2} \frac{1}{a} \alpha$ where $\tau_{2}^{\min }$ is the single root of $\quad \delta_{2} \frac{1}{a} \alpha \frac{\kappa \omega+\kappa^{2}}{\alpha \omega+\kappa \alpha+\kappa^{2}+2 \kappa \omega+\kappa \tau_{2}^{2}+\omega \tau_{2}^{2}}=\tau_{2}$ and $\frac{\left(\kappa^{2}-\omega \beta\right)}{a 2 \kappa}<(>) \tau_{1}^{*} \leq(\geq) 0$ if and only if $\kappa^{2}<(\geq) \omega \beta$. Furthermore, let $\tau_{1}^{*}\left(\tau_{0}\right)$ and $\tau_{2}^{*}\left(\tau_{0}\right)$ are given as the fixed points corresponding to $\bar{\tau}_{0}=\tau_{0}$ with the smallest absolute value. Then these functions will be continuous.

Proof. Notice first, that $\tau_{1}=f^{1}\left(\tau_{2}, \tau_{1}, \bar{\tau}_{0}\right)$ determines a third degree polynom in $\tau_{1}$, which is monotone increasing so it gives a single root for every $\tau_{0}$ and $\tau_{2}$. Similarly, $\tau_{2}=f^{2}\left(\tau_{2}, \tau_{1}, \bar{\tau}_{0}\right)$ also determines a monotone increasing third degree polynom in $\tau_{2}$ which gives a single unique root for every $\tau_{0}$ and $\tau_{1}$. It is also apparent that a marginal change in $\tau_{0}$ or $\tau_{2}$ in the first polynom or a marginal change in $\tau_{0}$ or $\tau_{1}$ in the second polynom will cause only a marginal change in the roots. This gives the continuity of $\tau_{1}^{*}\left(\tau_{0}\right)$ and $\tau_{2}^{*}\left(\tau_{0}\right)$.

For the existence, note that from the root of the polynom $\tau_{1}=f^{1}\left(\tau_{2}, \tau_{1}, \bar{\tau}_{0}\right), \tau_{1}^{2}\left(\tau_{2}^{2}\right)$ is a well defined continuous function. Therefore, the equilibrium is given by the fixed point of $\tau_{2}=\frac{1}{a}$ 
$\alpha \delta_{2} g^{2}\left(\tau_{2}\right)=f^{2}\left(\tau_{2}, \tau_{1}^{2}\left(\tau_{2}^{2}\right), \bar{\tau}_{0}\right)$ where $g(\cdot)$ continuously maps $\tau_{2}$ to the unite interval. As $\lim _{\tau_{2} \rightarrow 0} \frac{1}{a}$ $\alpha \delta_{2} g\left(\tau_{2}\right)=\delta_{2} \frac{1}{a} \alpha \frac{\kappa \omega+\kappa^{2}}{\tau_{1}^{2}(0) \kappa+\kappa^{2}+\tau_{1}^{2}(0) \omega+\tau_{0}^{2} \kappa+\alpha \kappa+\tau_{0}^{2} \omega+\alpha \omega+2 \kappa \omega}>0$, where $\tau_{1}^{2}(0)$ is finite and $0<\frac{1}{a} \alpha \delta_{2} g\left(\tau_{2}\right) \leq$ $\delta_{2} \frac{1}{a} \alpha$, there has to be a fixed point. The rest of the lemma comes from simple observation.

Lemma 2 The second order condition of the maximization problem in period 0 always holds, so the denominator of $f^{0}\left(\tau_{1}, \tau_{2}, \tau_{0}\right)$

$$
a\left(c_{1}^{2} \sigma_{y}+c_{1}^{2} s \sigma_{y} \sigma_{q}-c_{1}^{2} s \sigma_{y q}^{2}+\sigma_{q} b_{1}^{2}+2 c_{1} \sigma_{y q} b_{1}\right)>0
$$

Proof. Note that the matrix

$$
Q=\left(\left(\begin{array}{cc}
0 & 0 \\
0 & s
\end{array}\right)+\left(\begin{array}{cc}
\sigma_{y} & \sigma_{y q} \\
\sigma_{y q} & \sigma_{q}
\end{array}\right)^{-1}\right)^{-1}
$$

is positive definite as $s>0$. Consequently

$$
0<x Q x^{T}
$$

for all $x$. The lemma comes from the choice of

$$
\left.x=\left(\begin{array}{ll}
-a c_{1} d_{1} & \left(-a b_{1} d_{1}+s x_{i}\right)
\end{array}\right)-2\left(\begin{array}{cc}
\mu_{y} & \mu_{q}
\end{array}\right)\left(\begin{array}{cc}
0 & 0 \\
0 & \frac{1}{2} s
\end{array}\right)\right)
$$

as then

$$
0<x Q x^{T}=\left(\begin{array}{c}
\frac{a^{2}\left(c_{1}^{2} \sigma_{y}+c_{1}^{2} s \sigma_{y} \sigma_{q}-c_{1}^{2} s \sigma_{y q}^{2}+\sigma_{q} b_{1}^{2}+2 c_{1} \sigma_{y q} b_{1}\right)}{\sigma_{q} s+1} d_{1}^{2}- \\
-\frac{2 a c_{1} \sigma_{y q} s x_{i}-2 \sigma_{q} a b_{1} \mu_{q} s-2 a c_{1} \sigma_{y q} \mu_{q} s+2 \sigma_{q} a b_{1} s x_{i}}{\sigma_{q} s+1} d_{1} \\
+\frac{s^{2} \sigma_{q}\left(x_{i}-\mu_{q}\right)^{2}}{\sigma_{q} s+1}
\end{array}\right),
$$

which is possible for all $d_{1}$ only if

$$
a\left(c_{1}^{2} \sigma_{y}+c_{1}^{2} s \sigma_{y} \sigma_{q}-c_{1}^{2} s \sigma_{y q}^{2}+\sigma_{q} b_{1}^{2}+2 c_{1} \sigma_{y q} b_{1}\right)>0 .
$$

Proposition 6 There is always at least one equilibrium.

Proof. From Lemma 1, we have to show that the expression $\tau_{0}=g^{0}\left(\tau_{0}\right)=f^{0}\left(\tau_{2}^{*}\left(\tau_{0}\right), \tau_{1}^{*}\left(\tau_{0}\right), \tau_{0}\right)$ has at least one fix point. We proceed in 4 steps.

1. Note, that $g^{0}\left(\tau_{0}\right)=g^{0}\left(-\tau_{0}\right)$ for all $\tau_{0}$. It is so, because $\tau_{0}$ enters as $\tau_{0}^{2}$ to all building-blocks of $g^{0}\left(\tau_{0}\right)$.

2. We show that $\lim _{\tau_{0} \rightarrow \infty} g^{0}\left(\tau_{0}\right)=0$. Let us check the building-blocks separately. As $\tau_{0} \rightarrow 0$, $\tau_{2}^{*}$ goes to a constant, $\tau_{1}^{*}$ goes to 0 by the order of $\frac{1}{\tau_{0}^{2}}$, hence $c_{1}, \sigma_{y}, s$ goes to constants, $\sigma_{y q}$ and $b_{y}$ goes to 0 by the order of $\frac{1}{\tau_{0}^{2}}, \sigma_{q}$ goes to infinity by the order of $\tau_{0}^{4}$ and $b_{1}$ goes to 0 by 
the order of $\frac{1}{\tau_{0}^{2}}$. So the nominator of $g^{0}\left(\tau_{0}\right),\left(\sigma_{q} s+1\right)\left(c_{1}+b_{1}\right) b_{y}+s\left(1-b_{y}\right)\left(c_{1} \sigma_{y q}+b_{1} \sigma_{q}\right)$, goes to infinity by the order of $\tau_{0}^{2}$ from the speed of convergence of the term $\sigma_{q} s\left(c_{1} b_{y}+b_{1}\right)$. The denominator, $a\left(c_{1}^{2} \sigma_{y}+2 c_{1} \sigma_{y q} b_{1}+c_{1}^{2} s \sigma_{y} \sigma_{q}-c_{1}^{2} s \sigma_{y q}^{2}+\sigma_{q} b_{1}^{2}\right)$ also goes to infinity but by the order of $\tau_{0}^{4}$ given by the term of $c_{1}^{2} s \sigma_{y} \sigma_{q}$. Hence, $\lim _{\tau_{0} \rightarrow \infty} g^{0}\left(\tau_{0}\right)=0$.

3. The function $g^{0}\left(\tau_{0}\right)$ is continuous. It comes by the positivity of the denominator, which holds because of Lemma 2, and the continuity of all building-blocks.

4. Hence, $g^{0}\left(\tau_{0}\right)$ will cross the $45^{\circ}$ line necessarily, because it is symmetric, continuous and goes to 0 as $\tau_{0}$ increases without bound. Therefore, there will be a fixed point with $\tau_{0}^{*} \geq(<) 0$ if $g^{0}(0) \geq(<) 0$.

\section{A.4 Proof of Proposition 5}

Proof. The second half of the statement implies the first half as both the aggregate holdings and the volume are continuous functions of $\omega$. For the second half of the statement, it is sufficient to show that $\lim _{\omega \rightarrow \infty}\left|\frac{\tau_{0}^{n}}{\delta_{0}}-\frac{\tau_{1}^{n}}{\delta_{1}}\right|=\lim _{\omega \rightarrow \infty}\left|\frac{\tau_{0}^{n}}{\delta_{0}}\right|=\lim _{\omega \rightarrow \infty}\left|\frac{\tau_{1}^{n}}{\delta_{1}}\right| \rightarrow 0$ while $\left|\frac{\tau_{0}}{\delta_{0}}-\frac{\tau_{1}}{\delta_{1}}\right| \rightarrow C_{1},\left|\frac{\tau_{1}}{\delta_{1}}\right| \rightarrow C_{2}$ and $\left|\frac{\tau_{0}}{\delta_{0}}\right| \rightarrow C_{3}$, where $C_{1}, C_{2}$ and $C_{3}$ are non zero constants. In the no-announcement case, the equilibrium is characterized by the following equations:

$$
\begin{aligned}
\tau_{2}^{n} & =f^{2}\left(\tau_{2}^{n}, \tau_{1}^{n}, \tau_{0}^{n}\right)= \\
& =\frac{\delta_{2} \frac{1}{a} \alpha\left(\tau_{0}^{n 2} \omega+\tau_{1}^{n 2} \omega+\kappa \omega+\tau_{0}^{n 2} \kappa+\tau_{1}^{2} \kappa+\kappa^{2}\right)}{\tau_{0}^{n 2} \kappa+\tau_{0}^{n 2} \tau_{2}^{2}+\tau_{0}^{n 2} \omega+\alpha \tau_{0}^{n 2}+\alpha \tau_{1}^{n 2}+\alpha \omega+\kappa \alpha+\kappa^{2}+2 \kappa \omega+\kappa \tau_{2}^{n 2}+\omega \tau_{2}^{n 2}+\tau_{1}^{n 2} \tau_{2}^{n 2}+\tau_{1}^{n 2} \omega+\tau_{1}^{n 2} \kappa} \\
\tau_{1}^{n} & =f^{1}\left(\tau_{2}^{n}, \tau_{1}^{n}, \tau_{0}^{n}\right)= \\
& =\delta_{1} \tau_{2}^{2} \alpha \frac{\kappa^{2}}{a\left(\kappa \tau_{0}^{n 2} \tau_{2}^{2}+\kappa \omega \tau_{2}^{2}+\tau_{2}^{2} \kappa^{2}+\omega \tau_{1}^{n 2} \tau_{2}^{2}+2 \alpha \omega \tau_{2}^{2}+\kappa \tau_{1}^{n 2} \tau_{2}^{2}+2 \kappa \alpha \tau_{2}^{2}+\tau_{0}^{n 2} \omega \tau_{2}^{n 2}+\alpha \kappa^{2}+\kappa \omega \alpha\right)} \\
\tau_{0}^{n} & =f^{0}\left(\tau_{2}^{n}, \tau_{1}^{n}, \tau_{0}^{n}\right)=\delta_{0} \frac{b_{y}^{n}+\sigma_{q}^{n} s^{n}}{b_{1}^{n} a \sigma_{q}^{n}} .
\end{aligned}
$$

It is apparent, that for any $\tau_{1}^{n}, \tau_{0}^{n}, \lim _{\omega \rightarrow \infty} \tau_{2}^{n}$ is a finite, positive constant, and for any finite, positive $\tau_{2}^{n}$ and any $\tau_{0}^{n} \lim _{\omega \rightarrow \infty} \tau_{1}^{n}=0$. Consequently, $\lim _{\omega \rightarrow 0} \sigma_{q}^{n}=\lim _{\tau_{1} \rightarrow 0} \sigma_{q}^{n}=\infty$. Hence,

$$
\lim _{\omega \rightarrow \infty} \delta_{0} \frac{b_{y}^{n}+\sigma_{q}^{n} s^{n}}{b_{1}^{n} a \sigma_{q}^{n}}=\lim _{\omega \rightarrow \infty} \frac{s^{n}}{b_{1}^{n}}=\lim _{\omega \rightarrow \infty} \frac{\frac{b_{s}^{n}}{\left(\sigma_{s}^{n}+\frac{1}{\tau_{2}^{2}}\right)}}{b_{2}^{n} \frac{\left(b_{s}^{n}+e_{s}^{n}\right)}{b_{s}^{n}}+\frac{\bar{f}}{b_{s}}}=0
$$

, which holds because $\lim _{\omega \rightarrow \infty} b_{s}^{n}=0$, but $\lim _{\omega \rightarrow \infty} b_{2}^{n}, \lim _{\omega \rightarrow \infty} \sigma_{s}^{n}$ and $\lim _{\omega \rightarrow \infty} \frac{\left(b_{s}+e_{s}\right)}{b_{s}}=\lim _{\omega \rightarrow \infty} \frac{\tau_{1}^{2} \kappa^{2}+\alpha \kappa^{2}}{\alpha \kappa^{2}}=$ 1 are non zero constants.

In the announcement case, our equilibrium determining equations go to the following ones as 
$\omega \rightarrow \infty:$

$$
\begin{aligned}
\tau_{2} & =\delta_{2} \frac{1}{a} \alpha \frac{\left(\kappa+\tau_{0}^{2}+\tau_{1}^{2}\right)}{\left(\tau_{0}^{2}+2 \kappa+\tau_{2}^{2}+\tau_{1}^{2}+\alpha\right)} \\
\tau_{1} & =-\delta_{1} \tau_{2}^{2} \alpha \frac{\beta}{a\left(\tau_{1}^{2} \tau_{2}^{2}+\kappa \tau_{2}^{2}+\tau_{0}^{2} \tau_{2}^{2}+2 \alpha \tau_{2}^{2}+\kappa \alpha\right)} \\
\tau_{0} & =\frac{\delta_{0}\left(\sigma_{q} s+1\right)\left(c_{1}+b_{1}\right) b_{y}+s\left(1-b_{y}\right)\left(c_{1} \sigma_{y q}+b_{1} \sigma_{q}\right)}{a\left(c_{1}^{2} \sigma_{y}+c_{1}^{2} s \sigma_{y} \sigma_{q}-c_{1}^{2} s \sigma_{y q}^{2}+\sigma_{q} b_{1}^{2}+2 c_{1} \sigma_{y q} b_{1}\right)}
\end{aligned}
$$

where the building-blocks of the last equations are all of the corresponding limiting functions. By the observation of expressions for the building-blocks, it is apparent that all of them are going to finite, non-zero constants as $\omega \rightarrow \infty$. Hence, just by the same reasoning as in the existence theorem, there must be at least one equilibrium where all $\tau_{2}, \tau_{1}, \tau_{0}$ will be finite and non-zero. If $\lim _{\omega \rightarrow \infty} \frac{\tau_{1}}{\delta_{1}}$ and $\lim _{\omega \rightarrow \infty} \frac{\tau_{0}}{\delta_{1}}$ happened to be equal with certain combinations of the parameters, small perturbation on the parameters (for example perturbing $\delta_{0}$ ) would unambiguously make $\lim _{\omega \rightarrow \infty}\left|\frac{\tau_{0}}{\delta_{0}}-\frac{\tau_{1}}{\delta_{1}}\right|>0$. 